\title{
Extension of Pirogov-Sinai Theory of Phase Transitions to Infinite Range Interactions I. Cluster Expansion
}

\author{
Yong Moon Park \\ Department of Mathematics, Yonsei University, Seoul 120, Korea
}

\begin{abstract}
This paper is the first part of an extension of the Pirogov-Sinai theory of phase transitions at low temperatures, applicable to lattice systems with finite range interactions, to infinite range interactions. Transforming the systems to a version of an interacting contour model, we develop a cluster expansion. Making appropriate assumptions about the interactions, we prove that for sufficiently low temperatures the expansion converges and the cluster property holds.

In the sequel, we will use the cluster expansion method developed here to investigate the structure of a phase diagram for a given system. We will also give some applications of our results.
\end{abstract}

\section{Introduction}

The understanding of phase transitions remains one of the main objects in statistical physics. Since Peierls [14] invented an argument to show that the Ising model on $v$-dimensional lattice, $v \geqq 2$, with nearest neighbor ferromagnetic interactions has spontaneous magnetization at low temperatures, the argument has been made mathematically precise $[2,8]$ and extended in many directions, and new methods have been invented to prove coexistence of phases in various systems (see the references in $[16,18$ and 1]). Most methods require that the different phases are related by a symmetry of Hamiltonians and that the systems satisfy the reflection positivity $[4-7,16]$. An exception is the Pirogov-Sinai theory of phase transitions $[15,18]$ and its extensions $[1,3,9,19]$.

However, the Pirogov-Sinai theory (PS theory) and its extensions in the present forms are applicable only to classical (lattice) systems with finite range interactions. The main purpose in this paper and its sequel [13] is to extend the PS theory to classical lattice systems with infinite range interactions. The extension is meaningful for the following reasons: There are obviously many interesting lattice models with

\footnotetext{
* Work supported in part by the Korean Science Foundation
} 
infinite range interactions. On the other hand, one may hope that the PS theory can be eventually extended to particle systems interacting via (infinite range) two-body potentials in continuous $\mathbb{R}^{v}, v \geqq 2$.

The PS theory describing the behaviour of phase diagrams of lattice models was based on the notion of a contour model and a contour model with a parameter $[15,18]$. In [19], Zahradnik gave an alternative version of the PS theory without the use of the contour model with a parameter. Instead, he introduced a truncated contour model, not appearing in the usual PS theory. It turns out that Zahradnik's approach can be modified and improved to be applicable to our situation.

An intuitive reason why an extension of the PS theory to infinite range interactions should be possible may be given as follows: Consider the Hamiltonian given by interactions $\Phi_{A}, A \subset \mathbb{Z}^{v}$. Then, for any fixed positive real number $s \geqq 1$, the Hamiltonian $H_{\Lambda}$ on the finite region $\Lambda \subset \mathbb{Z}^{v}$ can be written as

$$
H_{\Lambda}=\sum_{\substack{A \subset A: \\ \operatorname{dam}(A) \leqq s}} \Phi_{A}+\sum_{\substack{A \subset \Lambda . \\ \operatorname{diam}(A)>s}} \Phi_{A}=H_{\Lambda, s}^{\prime}+H_{\Lambda, s}^{\prime \prime} .
$$

Suppose that we can choose $s \geqq 1$ in such a way that $H_{\Lambda, s}^{\prime}$ contains all the essential properties in the PS theory and that $H_{\Lambda, s}^{\prime \prime}$ is sufficiently smaller than $H_{\Lambda, s}^{\prime}$ in some sense. Then it is reasonable to expect that the PS theory for $H_{\Lambda}$ can be developed by means of an expansion method to control $H_{\Lambda, s}^{\prime \prime}$. The smallness of $H_{\Lambda, s}^{\prime \prime}$ may be insured by imposing some decay properties on the interaction $\Phi_{A}$. But we must be careful about the above reasoning, because the theory will be formulated in contour language. See the expression (1.1) and the discussion below.

Let us first discuss the general strategy of our extension. If interactions are finite range, there are no interactions between disjoint contours if one defines contours appropriately. This is the case in the usual PS theory $[15,18,19]$. However, if the interactions are infinite range, it is apparent that interactions between contours should be allowed. Introducing the notion of external contour systems [18] and stable ground states [19] we will transform the model to a version of an interacting contour model. The extension will be carried out in two stages: (a) Construction of pure phases for stable ground states and (b) Investigation of the full phase diagrams. In this paper we construct the pure phases for stable ground states by developing a cluster expansion method which turns out to be similar to a small activity expansion in statistical mechanics. For any stable ground state and for sufficiently low temperature, we show that the cluster expansion converges and that the cluster property holds. This implies that in the region of convergence the phase corresponding to stable ground states coexist and that they are pure states. In the sequel [13], we will use the cluster expansion method developed in this paper, and a modified and improved version of the truncated contour model method used by Zahradnik in [19] to investigate the structures of the phase diagrams. We will also give some applications of our result in Sect. 5 of part II [13].

In order to avoid unnecessary notational complications, we limit ourself to the systems with infinite range two-body interactions (plus arbitrary finite range interactions), which are translation invariant. Our results can be easily extended to a more general class of interactions without much difficulties. We believe that 
the results can also be extended to continuous lattice spin systems [3] and to Widom-Rowlinson models [1].

We now describe briefly the basic ideas, techniques and results on the derivation and on the proof of convergence of the cluster expansion, and outline the contents of this paper. We introduce the notion of contours, boundaries of configurations and external contour systems following [18 and 19] in Sect. 2.1. For any configuration $\omega$ for which its external boundary is given by an external $q$-contour system $\partial=\left\{\Gamma_{1}, \Gamma_{2}, \ldots, \Gamma_{n}\right\}$, we transform the Hamiltonian to a version of an interacting contour Hamiltonian:

$$
H(\partial ; \omega)=\sum_{\Gamma \in \hat{o}} \Phi_{1}(\Gamma ; \omega)+\sum_{\Gamma, \Gamma^{\prime} \in \hat{o}} \Phi_{2}\left(\Gamma, \Gamma^{\prime} ; \omega\right),
$$

where $\Phi_{1}$ and $\Phi_{2}$ are one and two body contour interactions respectively. For the details, see Sect. 2.2. In Sect. 2.3 we list assumptions on interactions (Peierls condition and regularity condition), and give definitions of stable ground states, partition function and contour correlation functions. In this way we transform the model to a version of an interacting contour model. The main result on the convergence and the cluster property of contour correlation functions is given in Theorem 2.3.4.

We derive the cluster expansion for contour correlation functions in Sects. 3.1-3.3. If the one-body contour interaction $\Phi_{1}$ in (1.1) satisfies the property in the PS theory (stability condition) and if the two-body contour interaction $\Phi_{2}$ is sufficiently smaller than $\Phi_{1}$ (regularity condition), an expansion method can be developed. We establish decoupling and recoupling identities, which will generate decoupling and recoupling processes respectively. We use the identities to derive the cluster expansion of the following type: Let $\Lambda \subset \mathbb{Z}^{v}$ be a bounded region and let $\rho_{\Lambda}(\partial)$ be correlation functions of external contour systems. Then

$$
\rho_{\Lambda}(\partial)=\sum_{\left\{\hat{\sigma}_{1}, \hat{\sigma}_{2}, \ldots, \hat{c}_{n}\right\}} K\left(\partial, \partial_{1}, \ldots, \partial_{n}\right) g_{\Lambda}\left(\partial \cup\left(\cup \partial_{i}\right)\right)
$$

where $g$ is a contour functional satisfying an integral equation of KirkwoodSalsburg type:

$$
g_{\Lambda}=1+\mathbf{K}_{\Lambda} g_{\Lambda}
$$

on a Banach space $\mathscr{F}$, where $\mathbf{K}_{\Lambda}$ is an operator on $\mathscr{F}$. Using the decoupling identity (3.1.8), and following a usual decoupling process similar to that in $[11,12]$, it is easy to obtain the expansion (1.2). However, contrary to standard cluster expansions $[11,12,16]$, the decoupling process is not enough for deriving the integral equation (1.3). Thus, in order to derive Eq. (1.3) and to control the operator $\mathbf{K}_{\Lambda}$, we use a new technique, namely the recoupling process.

In Sect. 4.1 we state the results on the convergence of the cluster expansion as $\Lambda$ tends to $\mathbb{Z}^{v}$ (Theorem 4.1.1) and on the cluster property (Theorem 4.1.2) which imply our main results. In Sect. 4.2, we establish some useful uniform bounds. Using the uniform bounds and a modification of the method used in [18], we prove convergence of the cluster expansion. In Sect. 4.4, we give a sketch of a proof of the cluster property. The appendix contains a proof of a technical lemma (Proposition 4.2.2.). 
Let us make one additional comment on some difficulties in this paper. Our extension is based on the use of the integral equation $(1.3)[16,12,18]$. The operator $\mathbf{K}_{\Lambda}$ in (1.3) is of the form:

$$
\mathbf{K}_{\Lambda}=\chi_{\Lambda} \mathbf{K} \chi_{\Lambda} \quad \mathbf{K}=\mathbf{K}_{1}+\mathbf{K}_{2}+\mathbf{K}_{3}+\mathbf{K}_{4} .
$$

The operators $\mathbf{K}_{2}$ and $\mathbf{K}_{3}$ defined in (3.3.18) and (3.3.19), respectively, have very complicated structures, which originate from the use of the recoupling process to derive (1.3). In the PS theory, these operators are absent. This paper becomes lengthy, because we have put a lot of effort into estimating the norms of $\mathbf{K}_{2}$ and $\mathbf{K}_{3}$.

In principle, it may be possible to derive a polymer type expansion $[17,11]$ by using decoupling and recoupling procedures used in this paper. Then our main results would follow from a method of combinatoric estimates $[10,11,17]$. Because of coding problems arising from the complicated expression of $\mathbf{K}$, we did not pursue this idea.

\section{Notations, Definitions and Main Results}

\subsection{Basic Notations, Contours and External Contour Systems}

We will follow the notations used in Sinai [18] and Zahradnik [19] very closely. Let $\mathbb{Z}^{v}$ be a $v$-dimensional lattice space $(v \geqq 2)$, and let $\Omega$ be a finite set of spin values. For any $\Lambda \subset \mathbb{Z}^{v}$, denote by $\Omega^{\Lambda}$ the set of all configurations on $\Lambda$. Throughout this paper we use the norm

$$
\|x\|=\max _{1 \leqq l \leqq v}\left|x_{i}\right|, \quad x \in \mathbb{Z}^{\nu} .
$$

Given $\Lambda \subset \mathbb{Z}^{v}$, we denote by bd $(\Lambda)$ the boundary of $\Lambda$ :

$$
\operatorname{bd}(\Lambda)=\left\{x \in \Lambda: \operatorname{dist}\left(x, \Lambda^{c}\right)=1\right\},
$$

where $\Lambda^{c}$ denotes the complement of $\Lambda$. A set $A \subset \mathbb{Z}^{v}$ is called connected if for any two points $x, x^{\prime} \in A$ there exists a sequence $x=x_{1}, x_{2}, \ldots, x_{n}=x^{\prime}$ of points $x_{i} \in A$ such that $\left\|x_{i}-x_{i-1}\right\|=1$ for $i=2, \ldots, n$. For each finite $\Lambda \subset \mathbb{Z}^{v}$ and each configuration $\omega \in \Omega^{\mathbb{Z}^{1}}$, denote by $\omega_{\Lambda}$ the restriction of $\omega$ on $\Lambda$.

Fix some family $Q \subset \Omega$ and a real number $s \geqq 1$. The family $Q$ and the real number $s \geqq 1$ will be chosen appropriately once a model is given. Given $q \in Q$, denote by $\omega_{q}$ the constant configuration $\omega(x)=q$ for all $x \in \mathbb{Z}^{v}$. We will call $\omega_{q}$, $q \in Q$, the ground states. For a ground state $\omega_{q}$ we say that a point $x \in \mathbb{Z}^{v}$ is a $q$-correct point of a configuration $\omega$ if for each $y \in \mathbb{Z}^{v}$ such that $\|x-y\| \leqq s, \omega(y)=q$. We say that $x \in \mathbb{Z}^{v}$ is a boundary point of a configuration $\omega$ if it is incorrect for each ground $q^{\prime} \in Q$. A boundary of a configuration $\omega$ is defined as a union of all boundary points, denoted by $\partial \omega$. A restriction $\omega$ to any finite connected component of $\partial \omega$ will be called a contour of a configuration $\omega$, and this component will be called the support of this contour. Thus a contour $\Gamma$ is given by $\Gamma=\left(M, \omega_{M}\right)$, where $M$ is the support of $\Gamma ; M=\operatorname{supp} \Gamma$. A contour is said to be finite if $\operatorname{card}(\operatorname{supp} \Gamma)<\infty$.

Let $\Gamma$ be a contour, $A$ component of $(\operatorname{supp} \Gamma)^{c}$ will be called a $q$-component if the neighboring spins of $\Gamma$ have the value $q$. If the exterior component of $(\operatorname{supp} \Gamma)^{c}$ 
is a $q$-component, then $\Gamma$ will be called $q$-contour and denoted by $\Gamma^{q}$. For any ground state $\omega_{q^{\prime}}, q^{\prime} \in Q$, denote by $\operatorname{Int}_{q^{\prime}} \Gamma$ the union of all interior $q^{\prime}$-components of $(\operatorname{supp} \Gamma)^{c}$. We write

$$
\begin{gathered}
\text { Int } \Gamma=\bigcup_{q^{\prime} \in Q} \operatorname{Int}_{q^{\prime}} \Gamma, \quad V(\Gamma)=\operatorname{supp} \Gamma \cup \operatorname{Int} \Gamma, \\
\operatorname{ext} \Gamma=(V(\Gamma))^{c}, \quad|\Gamma|=|\operatorname{supp} \Gamma|,
\end{gathered}
$$

where for any set $A \subset \mathbb{Z}^{v},|A|$ is the cardinality of $A$.

A finite connected subset $B \subset \mathbb{Z}^{v}$ will be called simple if $B^{c}$ has no interior components, i.e., $B^{c}$ is connected. For a given finite $\Lambda \subset \mathbb{Z}^{v}$ and a $q \in Q$, let

$$
\mathscr{C}_{q}(\Lambda)=\left\{\Gamma^{q}: V\left(\Gamma^{q}\right) \subset \Lambda, \quad \operatorname{dist}\left(V\left(\Gamma^{q}\right), \Lambda^{c}\right) \geqq 2\right\} .
$$

We emphasize that in the definition of $\mathscr{C}_{q}(\Lambda)$ we used the condition $V\left(\Gamma^{q}\right) \subset \Lambda$. Note, however, that if $\Lambda$ is simple, then $\mathscr{C}_{q}(\Lambda)$ is the family of all $q$-contours in $\operatorname{Int}(\Lambda)$. Let $\mathscr{C}_{q}\left(\mathbb{Z}^{v}\right)$ be the set of all finite $q$-contours. The family of all external $q$-contour systems is defined by

$$
\mathscr{E}_{q}(\Lambda)=\left\{\partial=\left\{\Gamma_{1}^{q}, \Gamma_{2}^{q}, \ldots, \Gamma_{n}^{q}\right\}: \Gamma_{i}^{q} \in \mathscr{C}_{q}(\Lambda), \quad \operatorname{dist}\left(V\left(\Gamma_{i}^{q}\right)\right), V\left(\Gamma_{j}^{q}\right) \geqq 2 \quad \text { if } \quad i \neq j\right\} .
$$

The empty set $\phi$ also belongs to $\mathscr{E}_{q}(\Lambda)$. We note that for a given $\partial \in \mathscr{E}_{q}(\Lambda)$ any contour $\Gamma \in \partial$ is external in the sence that $\Gamma \subset\left(V\left(\Gamma^{\prime}\right)\right)^{c}$ for any $\Gamma^{\prime} \in \partial$ if $\Gamma^{\prime} \neq \Gamma$. Let $\mathscr{E}_{q}\left(\mathbb{Z}^{v}\right)$ be the family of all $q$-contour systems consisting of finite $q$-contours.

For a given $q$-contour $\Gamma \in \mathscr{C}_{q}(\Lambda)$, denote by $\Omega(\Gamma)$ the set of all configurations $\omega_{V(\Gamma)}$ on $V(\Gamma)$, which satisfying the condition that being extended by $q$ to whole $\mathbb{Z}^{v}$, they have $\Gamma$ as one of its external $q$-contours.

Before closing this section we give a comment on the family of external $q$-contour systems $\mathscr{E}_{q}(\Lambda)$. For any bounded $\Lambda \subset \mathbb{Z}^{v}$ and $s \geqq 1$, denote

$$
\Lambda_{s}=\left\{x \in \Lambda: \operatorname{dist}\left(x, \Lambda^{c}\right)>s\right\} \text {. }
$$

Let $\Lambda \subset \mathbb{Z}^{v}$ be simple. Then, for any configuration $\omega$ with the property that $\omega(y)=q$ for any $y \in\left(\Lambda_{s}\right)^{c}$, there exists a unique maximal subset $\operatorname{ext}(\partial \omega)$ of $\partial \omega$, called the

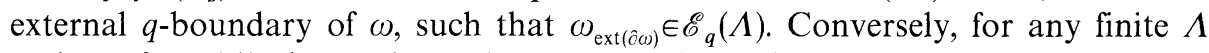
and any $\partial \in \mathscr{E}_{q}(\Lambda)$, there exists at least one configuration $\omega$ such that the restriction of $\omega$ to its external $q$-boundary coincides with $\partial$.

\subsection{Hamiltonians and Contour Hamiltonians}

In order to avoid unnecessary notational complications we mainly consider systems with infinite range two-body interactions plus arbitrary finite range interactions. Let some family $\left\{\Phi_{A}\right\}$ of finite range interactions (functions on $\Omega^{A}$ ) be given, invariant with respect to shifts in $\mathbb{Z}^{v}$ and with a finite interaction radius $s\left(\Phi_{A}=0\right.$ if $\operatorname{diam}(A)>s$ ). For any $x, y \in \mathbb{Z}^{v}$, let $J_{y-x}: \Omega \times \Omega \rightarrow \mathbb{R}$ be a symmetric function. For a finite $\Lambda \subset \mathbb{Z}^{v}$ and a configuration $\omega$, the Hamiltonian is given by

$$
H_{\Lambda}\left(\omega_{\Lambda} \mid \omega_{\Lambda^{c}}\right)=\sum_{\substack{A \wedge A \neq \phi: \\ d(A) \leq s}} \Phi_{A}\left(\omega_{A}\right)+\sum_{\{x, y\} \cap \Lambda \neq \phi} J_{x-y}(\omega(x), \omega(y)) .
$$


We will impose a decay property on the two-body interaction $J_{x-y}$ later.

Let $\Lambda$ be a simple, finite and connected region and let $\omega$ be a configuration such that its external boundary $\omega_{\text {ext }(\hat{c} \omega)}$ be given by an external $q$-contour system $\left\{\Gamma_{1}, \Gamma_{2}, \ldots, \Gamma_{n}\right\} \in \mathscr{E}_{q}(\Lambda)$. For any $B \subset \mathbb{Z}^{v}$, let $\omega_{B, q}$ denote the constant configuration on $B$ with its value $q \in Q$. Then

$$
H_{\Lambda}\left(\omega_{\Lambda} \mid \omega_{\Lambda^{c}, q}\right)=H\left(\omega_{\Lambda} \mid \omega_{\Lambda^{c}, q}\right)-H\left(\omega_{\Lambda, q} \mid \omega_{\Lambda^{c}, q}\right)+H\left(\omega_{\Lambda, q} \mid \omega_{\Lambda^{c}, q}\right),
$$

and

$$
\begin{aligned}
H_{\Lambda}\left(\omega_{\Lambda} \mid \omega_{A^{c}, q}\right)-H_{\Lambda}\left(\omega_{\Lambda, q} \mid \omega_{A^{c}, q}\right)= & \sum_{i=1}^{n} \sum_{A \subset V\left(\Gamma_{t}\right)}\left[\Phi_{A}\left(\omega_{A}\right)-\Phi_{A}\left(\omega_{A, q}\right)\right] \\
& +\sum_{i=1}^{n} \sum_{\{x, y\} \cap\left(\left(\bigcup_{j=1}^{n} V\left(\Gamma_{j}\right)\right) / V\left(\Gamma_{t^{\prime}}\right)\right)=\phi} \\
& \cdot\left[J_{x-y}(\omega(x), \omega(y))-J_{x-y}(q, q)\right] \\
& +\frac{1}{2} \sum_{i \neq j=1}^{n} \sum_{\substack{x \in V\left(\Gamma_{i}\right) \\
y \in V\left(\Gamma_{j}\right)}}\left[J_{x-y}(\omega(x), \omega(y))-J_{x-y}(q, q)\right] .
\end{aligned}
$$

Let $\omega\left(x ; V\left(\Gamma_{i}\right)\right)$ be the configuration defined by

$$
\omega\left(x ; V\left(\Gamma_{i}\right)\right)=\left\{\begin{array}{lll}
\omega(x) & \text { if } & x \in V\left(\Gamma_{i}\right) \\
q & \text { if } & x \notin V\left(\Gamma_{i}\right) .
\end{array}\right.
$$

Then from (2.2.3) it follows that

$$
H_{\Lambda}\left(\omega_{\Lambda} \mid \omega_{\Lambda^{c}, q}\right)-H_{\Lambda}\left(\omega_{\Lambda, q} \mid \omega_{\Lambda^{c}, q}\right)=\sum_{i=1}^{n} \Phi_{1}\left(\Gamma_{i} ; \omega\right)+\sum_{i \neq j=1}^{n} \Phi_{2}\left(\Gamma_{i}, \Gamma_{j} ; \omega\right)
$$

where for a given configuration $\omega$ with $\omega_{\text {ext }(\hat{\partial} \omega)}=\left\{\Gamma_{1}, \Gamma_{2}, \ldots, \Gamma_{n}\right\} \in \mathscr{E}_{q}(\Lambda), \Phi_{1}$ and $\Phi_{2}$ are defined by

$$
\begin{aligned}
\Phi_{1}\left(\Gamma_{i} ; \omega\right) \equiv & \sum_{A \subset V\left(\Gamma_{i}\right)}\left[\Phi_{A}\left(\omega_{A}\right)-\Phi_{A}\left(\omega_{A, q}\right)\right] \\
& +\sum_{\{x, y\} \cap V\left(\Gamma_{i}\right) \neq \phi}\left[J_{x-y}\left(\omega\left(x ; V\left(\Gamma_{i}\right)\right), \omega\left(y ; V\left(\Gamma_{i}\right)\right)\right)-J_{x-y}(q, q)\right]
\end{aligned}
$$

and

$$
\begin{aligned}
\Phi_{2}\left(\Gamma_{i}, \Gamma_{j} ; \omega\right) \equiv & -\frac{1}{2} \sum_{\substack{x \in V\left(\Gamma_{i}\right) \\
y \in V\left(\Gamma_{j}\right)}}\left[J_{x-y}(\omega(x), q)-J_{x-y}(q, q)\right] \\
& +\frac{1}{2} \sum_{\substack{x \in V\left(\Gamma_{i}\right) \\
y \in V\left(\Gamma_{j}\right)}}\left[J_{x-y}(\omega(x), \omega(y))-J_{x-y}(q, q)\right],
\end{aligned}
$$

respectively. We note that $\Phi_{1}(\Gamma ; \omega)$ depends only on the values of $\omega$ on $V(\Gamma)$. 
From (2.2.2)-(2.2.5) it follows that

$$
H_{\Lambda}\left(\omega_{\Lambda} \mid \omega_{\Lambda^{c}, q}\right)=\sum_{i=1}^{n} \Phi_{1}\left(\Gamma_{i} ; \omega\right)+\sum_{i \neq j=1}^{n} \Phi_{2}\left(\Gamma_{i}, \Gamma_{j} ; \omega\right)+H_{\Lambda}\left(\omega_{\Lambda, q} \mid \omega_{\Lambda^{c}, q}\right)
$$

Obviously the last term in the above does not depend on $\omega_{\Lambda}$. Therefore, discarding this term from $H_{\Lambda}\left(\omega_{\Lambda} \mid \omega_{\Lambda^{c}, q}\right)$ does not effect the conditional Gibbs densities. Thus for any configuration $\omega$ for which its external $q$-boundary is given by an external $q$-contour system $\partial=\left\{\Gamma_{1}, \Gamma_{2}, \ldots, \Gamma_{n}\right\} \in \mathscr{E}_{q}(\Lambda)$ we define the contour Hamiltonian by

$$
H(\partial ; \omega)=\sum_{\Gamma \in \partial} \Phi_{1}(\Gamma ; \omega)+\sum_{\Gamma, \Gamma^{\prime} \in \hat{\partial}} \Phi_{2}\left(\Gamma, \Gamma^{\prime} ; \omega\right),
$$

where $\Phi_{1}$ and $\Phi_{2}$ have been defined in (2.2.6) and (2.2.7) respectively.

\subsection{Assumptions, Definitions and Main Results}

Recall the definitions of the set of $q$-contours $\mathscr{C}_{q}(\Lambda)$ and the family of external $q$-contour systems $\mathscr{E}_{q}(\Lambda)$ in (2.1.4) and (2.1.5) respectively. For any finite $\Lambda \subset \mathbb{Z}^{v}$ and any $q \in Q$ we define the partition function by

$$
Z_{q}(\Lambda)=\sum_{\hat{c} \in \delta_{q}(\Lambda)} \prod_{\Gamma \in \hat{c}} \sum_{\omega_{V(\Gamma)} \in \Omega(\Gamma)} e^{-H(\hat{c}, \omega)}
$$

The term corresponding to the empty set $\partial=\phi$ in the above equals to 1 . Notice that for a simple $\Lambda$ the above definition coincides with the usual one corresponding to $q$-boundary conditions on $\left(\Lambda_{s}\right)^{c}$. For any $\Gamma \in \mathscr{C}_{q}\left(\mathbb{Z}^{v}\right)$, define the crystal partition function by

$$
Z_{q}(\Gamma)=\sum_{\omega \in \Omega(I)} e^{-\Phi_{1}(\Gamma, \omega)} .
$$

For $\Lambda=\operatorname{Int}_{q} \Gamma$, it follows from (2.3.1) that

$$
Z_{q}(\operatorname{Int} \Gamma)=\sum_{\hat{c} \in \mathcal{E}_{q}(\operatorname{Int} T)} \prod_{\Gamma^{\prime} \in \hat{o}} \sum_{\omega_{V(\Gamma)} \in \Omega\left(\Gamma^{\prime}\right)} e^{-H(\hat{c} ; \omega)} .
$$

For a given external $q$-contour system $\partial \in \mathscr{E}_{q}(\Lambda)$ we define the external $q$-contour correlation functions by

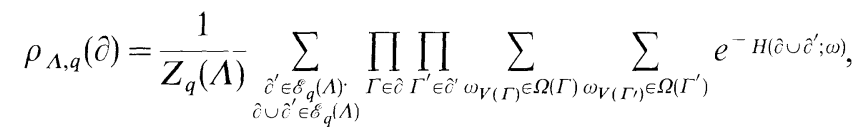

where the sum is taken over all $q$-contour systems $\partial^{\prime}$, compatible with $\partial$ in the sense that $\partial \cup \partial^{\prime} \in \mathscr{E}_{q}(\Lambda)$.

We now list assumptions on the interactions and then list our main results.

Assumption 2.3.1. [Peierls condition] For any $q$-contour $\Gamma=\left(M, \omega_{M}\right)$ let

$$
\begin{aligned}
\Phi(\Gamma)= & \sum_{A \cap M \neq \phi} \frac{|A \cap M|}{|A|}\left[\Phi_{A}\left(\omega_{A}\right)-\Phi_{A}\left(\omega_{A, q}\right)\right] \\
& +\sum_{\substack{\{x, y\} \cap M \neq \phi: \\
x-y \mid \leqq s}} \frac{|\{x, y\} \cap M|}{2}\left[J_{x-y}(\omega(x), \omega(y))-J_{x-y}(q, q)\right] .
\end{aligned}
$$


Then there exists a sufficiently large $\tau>0$ such that

$$
\Phi(\Gamma) \geqq \tau|\Gamma|
$$

holds for any $q$-contour $\Gamma$.

Since we have absorbed $\beta=1 / T$ into the Hamiltonian $H(\partial, \omega)$, the parameter $\tau$ is propositional to $\beta$. Next, we give an assumption on the decay property of the two-body potential.

Assumption 2.3.2. [Regularity condition] The two-body potential satisfies the bound

$$
\sup _{\omega}\left|J_{x-y}(\omega(x), \omega(y))\right| \leqq e^{-\delta(x, y)} J(\|x-y\|),
$$

where $\delta$ is a metric on $\mathbb{Z}^{v}$ such that

$$
\sup _{x} \sum_{y \in \mathbb{Z}^{2}} e^{-\delta(x, y)} \leqq M<\infty
$$

for some constant $M>0$, and $J: \mathbb{R} \rightarrow \mathbb{R}_{+}$is a non-negative function satisfying

$$
2 \sum_{r=0}^{\infty} \sum_{y \in \mathbb{Z}^{r}} J(2 s+r+\|y\|) \leqq \frac{1}{48} \tau,
$$

for the positive real number $s$ introduced in the definition of contours.

Remark. (a) If the two-body potential decays as

$$
\sup _{\omega}\left|J_{x-y}(\omega(x), \omega(y))\right| \leqq b\|x-y\|^{-(\lambda+\varepsilon)},
$$

for some $\lambda>2 v+1$ and $\varepsilon>v$, then one may choose the metric $\delta(x, y)$ as $\delta(x, y)=\varepsilon \log (1+\|x-y\|)$. In applications, $\tau$ is usually of the form $\tau=c \beta / s^{v}$ for some constant $c$ independent of $\beta$ and $s$. Note that we have absorbed $\beta$ into the Hamiltonian. In this case, (2.3.7) holds for sufficiently large $s$ or small $b$. See Appendix A of the sequel [13].

(b) Instead of the condition on the metric $\delta$ in (2.3.6), the following condition

$$
\delta(x, y) \rightarrow \infty \text { as } \quad\|x-y\| \rightarrow \infty
$$

is sufficient to get our main results (Theorem 2.3.4, Theorem 4.1.1 and Theorem 4.1.2). We impose the condition (2.3.6) stronger than (2.3.8) to investigate the structure of the phase diagrams.

Next, we define a contour functional $\Psi(\Gamma)$ by

$$
e^{-\Psi(\Gamma)} \equiv Z_{q}(\Gamma) / Z_{q}(\operatorname{Int} \Gamma) \text {. }
$$

As in [19], we introduce the notion of the stable ground states

Definition 2.3.3. A $q$-contour $\Gamma$ is called stable if

$$
\Psi(\Gamma) \geqq \frac{1}{3} \tau|\Gamma| .
$$

A ground state $q \in Q$ is called stable if every $\Gamma \in \mathscr{E}_{q}\left(\mathbb{Z}^{v}\right)$ is stable.

In the sequel [13] we will analyze the stability condition in detail. At present, we remark that if all ground states are related by a symmetry, then the Peierls 
condition and the regularity condition imply the stability. See for an instance Proposition 4.2 in Part II [13].

Theorem 2.3.4. Let $q \in Q$ be stable and assume that the regularity condition in Assumption 2.3.2 holds. Then for sufficiently larger $\tau$, the following results hold.

(a) The infinite volume limit

$$
\rho_{q}(\partial)=\lim _{\Lambda \rightarrow \mathbb{Z}^{\nu}} \rho_{\Lambda, q}(\partial)
$$

exists for any $\partial \in \mathscr{E}_{q}\left(\mathbb{Z}^{v}\right)$ as $\Lambda$ tends to $\mathbb{Z}^{v}$.

(b) The infinite volume limit contour correlation functions $\rho_{q}(\partial)$ satisfy the cluster property: For any $\partial, \partial^{\prime} \in \mathscr{E}_{q}\left(\mathbb{Z}^{v}\right)$ with $\partial \cup \partial^{\prime} \in \mathscr{E}_{q}\left(\mathbb{Z}^{v}\right)$,

$$
\rho_{q}\left(\partial \cup \partial^{\prime}\right)-\rho_{q}(\partial) \rho_{q}\left(\partial^{\prime}\right) \rightarrow 0
$$

as $\operatorname{dist}\left(\partial, \partial^{\prime}\right)$ tends to infinity.

In principle, it would be possible to estimate the decay rate of the clustering in terms of the metric $\delta$ by checking carefully each step in the proof of the above theorem in Sect. 4.4. Using the above result (and Proposition 2.1 in Part II) with the argument used in [18], one may construct a pure phase on $\Omega^{\mathbb{Z}^{*}}$ for each stable $q \in Q$. See the discussion given below Proposition 2.1 of Part II [13].

\section{The Cluster Expansion}

\subsection{Abbreviated Notations and Identities}

Before developing the cluster expansion, we introduce more abbreviated notations. We then state decoupling and recoupling identities which we will use to develop the cluster expansion.

From now on we fix a (stable) ground state $q \in Q$ and suppress $q$ from notations. We also write $\mathscr{C}\left(\mathbb{Z}^{v}\right)$ and $\mathscr{E}\left(\mathbb{Z}^{v}\right)$ as $\mathscr{C}$ and $\mathscr{E}$ respectively. For any $\Gamma \in \mathscr{C}$ and $\partial \in \mathscr{E}$, we write

$$
V(\partial)=\bigcup_{\Gamma \in \hat{\partial}} V(\Gamma), \quad \int_{\Gamma} d \omega=\sum_{\omega_{V(\Gamma)} \in \mathcal{S}(\Gamma)}, \quad \int_{\hat{\partial}} d \omega=\prod_{\Gamma \in \hat{\partial}} \int_{\Gamma} d \omega,
$$

and for any $\partial, \partial^{\prime} \in \mathscr{E}(\Lambda)$ with $\partial \cup \partial^{\prime} \in \mathscr{E}(\Lambda)$ we also write

$$
\Phi_{2}(\partial, \Gamma ; \omega)=\sum_{\Gamma^{\prime} \in \hat{c}} \Phi_{2}\left(\Gamma^{\prime}, \Gamma ; \omega\right), \quad \Phi_{2}\left(\partial, \partial^{\prime} ; \omega\right)=\sum_{\Gamma \in \hat{o}} \sum_{\Gamma^{\prime} \in \hat{\partial}^{\prime}} \Phi_{2}\left(\Gamma, \Gamma^{\prime} ; \omega\right) .
$$

Then it follows from (2.2.9) that

$$
H\left(\partial \cup \partial^{\prime} ; \omega\right)=H(\partial ; \omega)+H\left(\partial^{\prime} ; \omega\right)+\Phi_{2}\left(\partial, \partial^{\prime} ; \omega\right) .
$$

Using the notations in (3.1.1), and the definitions of partition function and contour correlation functions in (2.3.1) and (2.3.4) respectively, we may express that

and

$$
Z(\Lambda)=\sum_{\hat{o} \in \mathscr{E}(\Lambda)} \int_{\hat{o}} d \omega e^{-H(\hat{\sigma} ; \omega)}
$$

$$
\rho_{\Lambda}(\partial)=\frac{1}{Z(\Lambda)} \sum_{\left.\hat{o}^{\prime} \in \mathscr{E}(\Lambda \backslash V(\hat{c}))\right)} \int_{\hat{c}} d \omega \int_{\hat{\sigma}^{\prime}} d \omega e^{-H\left(\hat{\partial} \cup \tilde{\sigma}^{\prime}, \omega\right)} .
$$


From (3.1.3) it follows that

$$
e^{-H\left(\hat{\imath} \cup \hat{\sigma}^{\prime} ; \omega\right)}=e^{-H(\hat{\delta} ; \omega)} e^{-H\left(\hat{\sigma}^{\prime} ; \omega\right)} e^{-\Phi_{2}\left(\hat{\theta}, \hat{o}^{\prime} ; \omega\right)},
$$

for any $\partial, \partial^{\prime} \in \mathscr{E}$ with $\partial \cup \partial^{\prime} \in \mathscr{E}$.

Next, for any $\Gamma \in \mathscr{C}$ and $\partial \in \mathscr{E}$, denote

$$
g\left(\Gamma, \Gamma^{\prime} ; \omega\right)=e^{-\Phi_{2}\left(\Gamma, \Gamma^{\prime}, \omega\right)}-1, \quad g(\partial, \Gamma ; \omega)=e^{-\Phi_{2}(\hat{\partial}, \Gamma, \omega)}-1 .
$$

Then it follows that the decoupling identities

and

$$
e^{-\Phi_{2}\left(\hat{o}, \hat{o}^{\prime}, \omega\right)}=\prod_{\Gamma^{\prime} \in \hat{o}^{\prime}}\left[\left(e^{-\Phi_{2}\left(\hat{c}, \Gamma^{\prime}, \omega\right)}-1\right)+1\right]=1+\sum_{\phi \neq \hat{o}_{1} \subset \hat{o}^{\prime}} \prod_{\Gamma^{\prime} \in \hat{o}_{1}} g\left(\partial, \Gamma^{\prime} ; \omega\right)
$$

$$
g_{\Lambda}\left(\partial, \Gamma^{\prime} ; \omega\right)=\prod_{\Gamma \in \hat{\imath}}\left[e^{-\Phi_{2}\left(\Gamma, \Gamma^{\prime} ; \omega\right)}-1+1\right]-1=\sum_{\phi \neq \hat{o}_{1} \subset \hat{0}}\left(\prod_{\Gamma \in \hat{c}_{1}} g\left(\Gamma, \Gamma^{\prime} ; \omega\right)\right)
$$

hold for any $\partial, \partial^{\prime} \in \mathscr{E}$ with $\partial \cup \partial^{\prime} \in \mathscr{E}$. On the other hand, it also follows that the recoupling identity

$$
\begin{aligned}
1-e^{-\Phi_{2}\left(\hat{\theta}, \hat{\sigma}^{\prime}: \omega\right)}= & 1-\prod_{\Gamma^{\prime} \in \hat{\sigma}^{\prime}} e^{-\Phi_{2}\left(\hat{\theta}, \Gamma^{\prime}: \omega\right)}=\prod_{\Gamma^{\prime} \in \hat{\sigma}^{\prime}}\left[\left(1-e^{-\Phi_{2}\left(\hat{\theta}, \Gamma^{\prime} ; \omega\right)}\right)+e^{-\Phi_{2}\left(\hat{\theta}, \Gamma^{\prime} ; \omega\right)}\right] \\
& -\prod_{\Gamma^{\prime} \in \hat{o}^{\prime}} e^{-\Phi_{2}\left(\hat{\theta}, \Gamma^{\prime} ; \omega\right)}=\sum_{\phi \neq \hat{\sigma}_{1}^{\prime} \subset \hat{o}^{\prime}}\left(\prod_{\Gamma^{\prime} \in \hat{\sigma}_{1}^{\prime}}(-1) g\left(\partial, \Gamma^{\prime} ; \omega\right)\right) e^{-\Phi_{2}\left(\hat{c}, \hat{\sigma}^{\prime}-\hat{\sigma}_{1}^{\prime} ; \omega\right)},
\end{aligned}
$$

holds for any $\partial, \partial^{\prime} \in \mathscr{E}$ with $\hat{\partial} \cup \partial^{\prime} \in \mathscr{E}$.

In order to simplify notation and expressions further, we will use the following conventions:

(a) Whenever we use an expression like $F\left(\partial_{1}, \ldots, \partial_{n} ; \omega\right)$ for given external contour systems $\left\{\partial_{1}, \ldots, \partial_{n}\right\}$, it means that $F$ depends only on configurations $\omega$ in $\Omega\left(\cup \partial_{i}\right)$, where

$$
\Omega\left(\cup \partial_{i}\right)=\bigcup_{I \in \cup \hat{c}_{\imath}} \Omega(\Gamma) .
$$

(b) We will use the abused expressions such as

$$
F(\partial ; \omega)=\sum_{\partial^{\prime}} \int_{\hat{c}^{\prime}} d \omega \bar{F}\left(\partial, \partial^{\prime} ; \omega\right) .
$$

The above really means that $F(\partial ; \omega)$ is given by

$$
F(\partial ; \omega)=\sum_{\hat{o}^{\prime}} \int_{\partial^{\prime}} d \omega^{\prime} \bar{F}\left(\partial, \partial^{\prime} ; \omega \cup \omega^{\prime}\right) .
$$

Thus, $F(\partial ; \omega)$ in (3.1.12) is still dependent on $\omega \in \Omega(\partial)$.

Throughout this paper and the sequel [13] we will use the above conventions without mentioning them explicitly.

\subsection{The Cluster Expansion}

Let $B \subset \mathbb{Z}^{v}$ be a finite subset. For any external $q$-contour system $\partial, \partial^{\prime} \in \mathscr{E}(B)$ and for any configuration $\omega$ for which $\partial \cup \partial^{\prime}$ is the external q-boundary of $\omega$ we define

$$
f_{B}(\partial, \omega)=\sum_{\hat{c}^{\prime} \in \mathscr{f}(B \backslash V(\hat{\sigma}))} \int_{\hat{c}^{\prime}} d \omega e^{-H\left(\hat{o} \cup \hat{\sigma}^{\prime} ; \omega\right)} .
$$


Using (3.1.6), the decoupling identity (3.1.8), writing $\partial^{\prime}=\partial_{1} \cup \partial^{\prime \prime}$ and summing over $\partial^{\prime \prime}$, we obtain

$$
f_{B}(\partial ; \omega)=e^{-H(\hat{c} ; \omega)} \sum_{\hat{c}_{1} \in \mathscr{\delta}(B \backslash V(\hat{\partial}))} \int_{\hat{c}_{1}} d \omega\left(\prod_{\Gamma \subset \hat{c}_{1}} g(\partial, \Gamma ; \omega)\right) f_{B \backslash V(\hat{c})}\left(\partial_{1}, \omega\right),
$$

where the term in the sum corresponding to $\partial_{1}=\phi$ is allowed. Notice that

$$
Z(B \backslash V(\partial))=f_{B \backslash V(\hat{)})}(\phi ; \omega)
$$

by (3.1.4) and (3.2.1).

For a simplification of notation, denote

$$
\bar{K}(\partial, \omega) \equiv e^{-H(\hat{\partial} ; \omega)}, \quad \bar{K}\left(\partial, \partial^{\prime} ; \omega\right) \equiv e^{-H(\hat{c}, \omega)} \prod_{\Gamma \in \hat{o}^{\prime}} g(\partial, \Gamma ; \omega) .
$$

From (3.2.2) we have

$$
f_{B}(\partial ; \omega)=\bar{K}(\partial ; \omega) Z(B \backslash V(\partial))+\sum_{\phi \neq \hat{c}_{1} \in \delta(B \backslash V(\hat{o}))} \int_{\hat{c}_{1}} d \omega \bar{K}\left(\partial, \partial_{1} ; \omega\right) f_{B \backslash V(\hat{\partial})}\left(\partial_{1} ; \omega\right) .
$$

Iterating (3.2.5) we obtain

$$
\begin{aligned}
f_{B}(\hat{\partial} ; \omega)= & \bar{K}(\partial ; \omega) Z(B \backslash V(\partial)) \\
& +\sum_{n=1}^{\infty}\left(\sum_{\left\{\hat{o}_{1}, \hat{\partial}_{2}, \ldots, \hat{o}_{n}\right\}}^{\prime} \prod_{i=1}^{n} \int_{\hat{c}_{i}} d \omega \bar{K}\left(\partial, \partial_{1} ; \omega\right) \ldots \bar{K}\left(\partial_{n-1}, \partial_{n} ; \omega\right) \bar{K}\left(\partial_{n} ; \omega\right)\right) \\
& \cdot Z\left(\Lambda \backslash V\left(\partial \cup\left(\bigcup_{i=1}^{n} \partial_{i}\right)\right)\right)
\end{aligned}
$$

where in the sum $\Sigma^{\prime}$ the summation is over all $\left\{\partial_{1}, \partial_{2}, \ldots, \partial_{n}\right\}$ satisfying $\cup \partial_{i} \in \mathscr{E}(B \backslash V(\partial)), \partial_{i} \neq \phi$ and $V\left(\partial_{i}\right) \cap V\left(\partial_{j}\right)=\phi$ if $i \neq j$.

We write

$$
\begin{aligned}
K(\partial ; \omega) & \equiv \bar{K}(\partial ; \omega) / \prod_{\Gamma \in \hat{\partial}} Z(\operatorname{Int} \Gamma), \quad \tilde{K}\left(\partial, \partial^{\prime} ; \omega\right) \equiv \bar{K}\left(\partial, \partial^{\prime} ; \omega\right) / \prod_{\Gamma \in \hat{c}} Z(\operatorname{Int} \Gamma), \\
K\left(\partial_{1}, \partial_{2}, \ldots, \partial_{n} ; \omega\right) & \equiv \tilde{K}\left(\partial_{1}, \partial_{2} ; \omega\right) \ldots \tilde{K}\left(\partial_{n-1}, \partial_{n} ; \omega\right) K\left(\partial_{n} ; \omega\right) \\
K\left(\partial_{1}, \partial_{2}, \ldots, \partial_{n}\right) & =\prod_{i=1}^{n} \int_{\partial_{i}} d \omega K\left(\partial_{1}, \ldots, \partial_{n} ; \omega\right)
\end{aligned}
$$

and

$$
g_{\Lambda}(\partial) \equiv \frac{Z(\Lambda \backslash V(\partial)) \prod_{\Gamma \in \hat{\partial}} Z(\operatorname{Int} \Gamma)}{Z(\Lambda)}-
$$

Then from (3.2.6) it follows that

$$
\begin{aligned}
f_{\Lambda}(\partial ; \omega)= & K(\partial ; \omega) g_{\Lambda}(\partial) Z(\Lambda) \\
& +\sum_{n=1}^{\infty}\left(\sum_{\left\{\hat{o}_{1}, \ldots, \hat{c}_{n}\right\}}^{\prime} \prod_{i=1}^{n} \int_{\partial_{i}} d \omega K\left(\partial, \hat{\partial}_{1}, \ldots \partial_{n} ; \omega\right)\right) \\
& \cdot g_{\Lambda}\left(\partial \cup\left(\bigcup_{i=1}^{n} \partial_{i}\right)\right) Z(\Lambda) .
\end{aligned}
$$


Since

$$
\rho_{\Lambda}(\partial)=\int_{\partial} d \omega f_{\Lambda}(\partial ; \omega) / Z(\Lambda)
$$

we obtain from (3.2.9) that

$$
\rho_{\Lambda}(\partial)=K(\partial) g_{\Lambda}(\partial)+\sum_{n=1}^{\infty} \sum_{\left\{\hat{c}_{1}, \hat{o}_{2}, \ldots, \hat{o}_{n}\right\}}^{\prime} K\left(\partial, \partial_{1}, \ldots, \partial_{n}\right) g_{\Lambda}\left(\partial \cup\left(\bigcup_{i=1}^{n} \partial_{i}\right)\right),
$$

where in the sum $\Sigma^{\prime}$ the summation is over all $\left\{\partial_{1}, \partial_{2}, \ldots, \partial_{n}\right\}$ satisfying $\cup \partial_{i} \in \mathscr{E}(\Lambda \backslash V(\partial)), \partial_{i} \neq \phi$ and $V\left(\partial_{i}\right) \cap V\left(\partial_{j}\right)=\phi$ if $i \neq j$. The above expression is called the cluster expansion.

Let us express the decoupling process (3.2.9) and the cluster expansion (3.2.10) into more compact forms. For any $\partial, \partial^{\prime} \in \mathscr{E}\left(\mathbb{Z}^{v}\right)$ with $\partial \cup \partial^{\prime} \in \mathscr{E}\left(\mathbb{Z}^{v}\right)$ with $\partial \cup \partial^{\prime} \in \mathscr{E}\left(\mathbb{Z}^{v}\right)$ we define

$$
A\left(\partial^{\prime},(\partial ; \omega)\right)= \begin{cases}K(\partial ; \omega) & \text { if } \partial^{\prime}=\phi \\ \sum_{\left\{\partial_{1}, \ldots, \hat{\sigma}_{n}\right\} ;} \prod_{i=1}^{n} \int_{\partial_{i}} d \omega K\left(\partial, \partial_{1}, \ldots, \partial_{n} ; \omega\right) & \text { if } \partial^{\prime} \neq \phi . \\ \cup \partial_{i}=\partial^{\prime}, \partial_{i} \neq \phi, & \\ V\left(\partial_{i}\right) \cap V\left(\partial_{j}\right)=\phi & \end{cases}
$$

Then the expression (3.2.9) can be written as

$$
f_{\Lambda}(\partial ; \omega)=\sum_{\hat{\sigma}^{\prime} \in \mathcal{\delta}(\Lambda \backslash V(\hat{c}))} A\left(\partial^{\prime},(\partial ; \omega)\right) g_{\Lambda}\left(\partial \cup \partial^{\prime}\right) Z(\Lambda) .
$$

If we define

$$
A\left(\partial^{\prime}, \partial\right) \equiv \int_{\hat{\partial}} d \omega A\left(\partial^{\prime},(\partial ; \omega)\right)
$$

then the cluster expansion also can be written into the compact form

$$
\begin{aligned}
\rho_{\Lambda}(\partial) & =\sum_{\partial^{\prime} \in \delta(\Lambda \backslash V(\hat{c}))} \int_{\hat{c}} d \omega A\left(\partial^{\prime},(\partial ; \omega)\right) g_{\Lambda}\left(\partial \cup \partial^{\prime}\right) \\
& =\sum_{\partial^{\prime} \in \delta(\Lambda \backslash V(\hat{\partial}))} A\left(\partial^{\prime}, \partial\right) g_{\Lambda}\left(\partial \cup \partial^{\prime}\right) .
\end{aligned}
$$

The expressions in (3.2.12) and (3.2.14) will be called the decoupling process and the cluster expansion respectively.

\subsection{An Integral Equation of a Kirkwood-Salsburg Type}

We now derive an integral equation of a Kirkwood-Salsburg type of the following form for the external boundary functional $g_{\Lambda}(\partial)$ defined in (3.2.8):

$$
g_{\Lambda}=1+\mathbf{K}_{\Lambda} g_{\Lambda}
$$

on a Banach space $\mathscr{F}$, where $\mathbf{K}_{\Lambda}$ is an operator on $\mathscr{F}$. As mentioned in the introduction, we will use a recoupling process generated by the recoupling identity (3.1.10). It may be possible to derive an integral equation of type (3.3.1) by using only the decoupling process (3.2.9) (or (3.2.12)). Then one would have some difficulties in the estimation of the norm of $\mathbf{K}_{\Lambda}$. We believe that, in order to derive 
the integral equation (3.3.1) and to control the operator $\mathbf{K}_{\Lambda}$ in (3.3.1) simultaneously, it seems that the use of the recoupling identity (3.1.10) is unavoidable.

Let us start to derive an integral equation for $g_{\Lambda}$. For any $\Gamma_{0} \in \mathscr{C}$, let $\left[\Gamma_{0}\right]$ be the family of external contour systems defined by

$$
\left[\Gamma_{0}\right]=\left\{\Gamma \in \mathscr{C}: \operatorname{dist}\left(\operatorname{supp} \Gamma_{0}, V(\Gamma)\right) \leqq 1\right\} .
$$

Then, from the definition of $Z(B)$ in (3.1.4) it is easy to check that for any given $\Gamma_{0} \in \mathscr{E}(B)$

$$
Z(B)=Z\left(\left(B \backslash V\left(\Gamma_{0}\right)\right) \cup \operatorname{Int} \Gamma_{0}\right)+\sum_{\substack{\Gamma \in\left[\Gamma_{0}\right] \\ I \in \mathscr{C}(B)}} \sum_{\hat{c}^{\prime} \in \mathcal{E}(B \backslash V(\Gamma))} \int_{\Gamma} d \omega \int_{\hat{\sigma}^{\prime}} d \omega e^{-H\left(\Gamma \cup \hat{c}^{\prime} ;()\right)},
$$

and

$$
\begin{aligned}
Z\left(\left(B \backslash V\left(\Gamma_{0}\right)\right) \cup \operatorname{Int} \Gamma_{0}\right) & =\sum_{\substack{\hat{o} \in \mathscr{E}\left(\left(B \backslash V\left(\Gamma_{0}\right)\right) \cup \operatorname{Int} \Gamma_{0}\right)\\
}} d \omega e^{-H(\hat{c}, \omega)} \\
& =\sum_{\substack{\hat{c}^{\prime} \in \mathcal{E}\left(B \backslash V\left(\Gamma_{0}\right)\right) \\
\hat{c}^{\prime \prime} \in \mathcal{\delta}\left(\operatorname{Int} \Gamma_{0}\right)}} \int_{\hat{c}^{\prime}} d \omega \int_{\hat{\iota}^{\prime \prime}} d \omega e^{-H\left(\hat{c}^{\prime} \cup \hat{c}^{\prime \prime} \cdot(\omega)\right.} .
\end{aligned}
$$

For a finite $B \subset \mathbb{Z}^{v}$ and $\Gamma_{0} \in \mathscr{C}(B)$, write

$$
\begin{aligned}
Z\left(B \backslash V\left(\Gamma_{0}\right)\right) Z\left(\operatorname{Int} \Gamma_{0}\right)= & {\left[Z\left(B \backslash V\left(\Gamma_{0}\right)\right) Z\left(\operatorname{Int} \Gamma_{0}\right)-Z\left(\left(B \backslash V\left(\Gamma_{0}\right)\right) \cup \operatorname{Int} \Gamma_{0}\right)\right] } \\
& +Z\left(\left(B \backslash V\left(\Gamma_{0}\right)\right) \cup \operatorname{Int} \Gamma_{0}\right) .
\end{aligned}
$$

We will estimate the first in the right-hand side in the above by using the recoupling identity (3.1.10).

Let $B \subset \mathbb{Z}^{v}$ and $\Gamma_{0} \in \mathscr{E}(B)$. For given $\hat{o}_{1}=\left\{\Gamma_{1}, \Gamma_{2}, \ldots, \Gamma_{n}\right\} \in \mathscr{E}\left(\operatorname{Int} \Gamma_{0}\right)$ and $\partial_{1}^{\prime} \in \mathscr{E}\left(B \backslash V\left(\Gamma_{0}\right)\right)$, denote

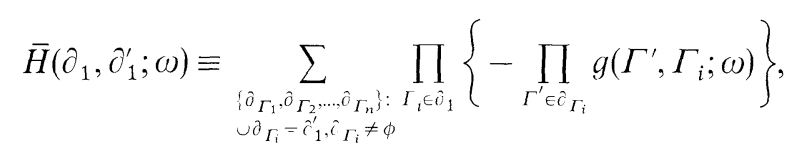

where in the sum it can be happen that $\partial_{\Gamma_{t}} \cap \partial_{\Gamma_{j}} \neq \phi$. Then, using the recoupling identity (3.1.10) and (3.1.9) we obtain that for any $\partial^{\prime \prime} \in \mathscr{E}\left(\operatorname{Int} \Gamma_{0}\right), \partial^{\prime} \in \mathscr{E}\left(B \backslash V\left(\Gamma_{0}\right)\right)$,

$$
1-e^{-\Phi_{2}\left(\hat{c}^{\prime}, \hat{c}^{\prime \prime}, \omega\right)}=\sum_{\phi \neq \hat{\partial}_{1} \subset \hat{c}^{\prime \prime} \hat{c}_{1}^{\prime} \subset \hat{c}^{\prime}} \vec{H}\left(\partial_{1}, \partial_{1}^{\prime} ; \omega\right) e^{-\Phi_{2}\left(\hat{c}^{\prime} \cdot \hat{c}^{\prime \prime}-\hat{o}_{1}, \omega\right)}
$$

We use (3.3.4), (3.3.7) and the fact that $H\left(\partial^{\prime \prime} ; \omega\right)=H\left(\partial_{1}\right)+H\left(\partial^{\prime \prime}-\partial_{1}\right)+$ $\Phi_{2}\left(\partial_{1}, \partial^{\prime \prime}-\partial_{1} ; \omega\right)$ by (3.1.3), and we then write $\partial^{\prime}=\partial_{1}^{\prime} \cup \partial_{1}^{\prime \prime}$ and sum over $\partial_{1}^{\prime \prime}$ to obtain

$$
\begin{aligned}
& Z\left(B \backslash V\left(\Gamma_{0}\right)\right) Z\left(\operatorname{Int} \Gamma_{0}\right)-Z\left(\left(B \backslash V\left(\Gamma_{0}\right)\right) \cup \operatorname{Int} \Gamma_{0}\right) \\
& =\sum_{\substack{\left.\hat{\sigma}^{\prime} \in \mathscr{E}\left(B \backslash V\left(\Gamma_{0}\right)\right)\right) \\
\hat{c}^{\prime \prime} \in \mathscr{E}\left(\ln \Gamma_{0}\right)}} \int_{\hat{c}^{\prime}} d \omega e^{-H\left(\hat{o}^{\prime}, \omega\right)} e^{-H\left(\hat{c}^{\prime \prime}, \omega\right)}\left[1-e^{-\Phi_{2}\left(\hat{c}^{\prime}, \hat{c}^{\prime \prime} ; \omega\right)}\right] \\
& =\sum_{\phi \neq \hat{c}^{\prime \prime} \in \mathscr{E}\left(\operatorname{Int} \Gamma_{0}\right)} \sum_{\phi \neq \hat{\sigma}_{1} \subset \hat{c}^{\prime \prime}} \sum_{\hat{c}_{1}^{\prime} \in \mathscr{E}\left(B \backslash V\left(\Gamma_{0}\right)\right)} \int_{\hat{c}^{\prime \prime}} d \omega \int_{\hat{c}_{1}} \int_{\hat{c}_{1}^{\prime}} d \omega \bar{H}\left(\partial_{1}, \partial_{1}^{\prime} ; \omega\right) \\
& \cdot e^{-H\left(\hat{c}_{1}, \omega\right)} e^{-\Phi_{1}\left(\hat{o}^{\prime \prime}-\hat{c}_{1} ; \omega\right)} \tilde{f}_{B \backslash V\left(\Gamma_{1}\right)}\left(\left(\partial^{\prime \prime}-\partial_{1}\right) \cup \partial_{1}^{\prime} ; \omega\right),
\end{aligned}
$$


where

$$
\tilde{f}_{B \backslash V\left(\Gamma_{1}\right)}\left(\left(\partial^{\prime \prime}-\partial_{1}\right) \cup \partial_{1}^{\prime} ; \omega\right)=\sum_{\hat{\sigma}_{1}^{\prime \prime} \in \mathscr{E}\left(B \backslash V\left(\Gamma_{0} \cup \hat{\sigma}_{1}^{\prime}\right)\right) \hat{\partial}_{1}^{\prime \prime}} d \omega e^{-H\left(\left(\hat{\partial}^{\prime \prime}-\partial_{1}\right) \cup\left(\hat{\sigma}_{1}^{\prime} \cup \hat{\sigma}_{1}^{\prime \prime}\right), \omega\right)} .
$$

Let us denote

$$
G\left(\partial_{1}, \partial_{2} ; \omega\right) \equiv \prod_{\Gamma_{1} \in \hat{\sigma}_{2}} g\left(\partial_{1}, \Gamma_{2} ; \omega\right)
$$

Then by the decoupling identity (3.1.8)

$$
e^{-\Phi_{2}\left(\partial_{1}, \hat{\partial}_{2} ; \omega\right)}=\sum_{\partial_{2}^{\prime} \in \hat{\theta}_{2}} G\left(\partial_{1}, \partial_{2}^{\prime} ; \omega\right)
$$

where the term corresponding to $\partial_{2}^{\prime}=\phi$ equals to 1 . Substituting (3.3.11) with $\partial_{2}=\partial^{\prime \prime}-\partial_{1}$ into (3.3.8), writing $\partial^{\prime \prime}-\partial_{1}=\partial_{2}^{\prime} \cup \partial_{2}^{\prime \prime}$ and summing over $\partial_{2}^{\prime \prime}$ we obtain from (3.3.8) (and the expression of $f_{B}(\partial ; \omega)$ in $(3.2 .1)$ ) that

$$
\begin{aligned}
Z\left(B \backslash V\left(\Gamma_{0}\right)\right) Z\left(\operatorname{Int} \Gamma_{0}\right)-Z\left(\left(B \backslash V\left(\Gamma_{0}\right)\right) \cup \operatorname{Int} \Gamma_{0}\right) \\
=\sum_{\substack{\hat{o}_{1} \cup \hat{o}_{2}^{\prime} \in \mathscr{E}^{\prime}\left(\operatorname{Int} \Gamma_{0}\right) \cdot \hat{o}_{1}^{\prime} \in \mathscr{E}\left(B \backslash V\left(\Gamma_{0}\right)\right) \\
\partial_{1} \neq \phi}} \sum_{\partial_{1}} d \omega \int_{\hat{o}_{2}^{\prime}} d \omega \int_{\hat{\partial}_{1}^{\prime}} d \omega \\
\quad \cdot \bar{H}\left(\partial_{1}, \partial_{1}^{\prime} ; \omega\right) G\left(\partial_{1}, \partial_{2}^{\prime} ; \omega\right) e^{-H\left(\hat{o}_{1} ; \omega\right)} f_{\left(B \backslash V\left(\Gamma_{0}\right)\right) \cup\left(\operatorname{Int} \Gamma_{0} \backslash V\left(\hat{o}_{1}\right)\right)}\left(\partial_{1}^{\prime} \cup \partial_{2}^{\prime} ; \omega\right),
\end{aligned}
$$

where the case for $\partial_{2}^{\prime}=\phi$ is allowed.

Next, from the definition of $f_{B}(\partial, \omega)$ in (3.2.1) it follows that

$$
\begin{aligned}
& f_{\left(B \backslash V\left(\Gamma_{0}\right)\right) \cup\left(\operatorname{Int} \Gamma_{0} \backslash V\left(\partial_{1}\right)\right)}\left(\partial_{1}^{\prime} \cup \partial_{2}^{\prime} ; \omega\right) \\
& \quad=f_{B \backslash V\left(\partial_{1}\right)}\left(\partial_{1}^{\prime} \cup \partial_{2}^{\prime} ; \omega\right)-\sum_{\Gamma \in\left[\Gamma_{0}\right]} f_{B \backslash V\left(\hat{\sigma}_{1}\right)}\left(\{\Gamma\} \cup \partial_{1}^{\prime} \cup \partial_{2}^{\prime} ; \omega\right) .
\end{aligned}
$$

Using (3.2.12) (with $\Lambda=B \backslash V\left(\partial_{1}\right)$ ) and (3.3.13) we obtain the following relation:

$$
\begin{aligned}
& f_{\left(B \backslash V\left(\Gamma_{0}\right)\right) \cup\left(\operatorname{Int} \Gamma_{0} \backslash V\left(\tilde{c}_{1}\right)\right)}\left(\partial_{1}^{\prime} \cup \partial_{2}^{\prime} ; \omega\right) \\
& =\sum_{\hat{\sigma}^{\prime} \in \mathscr{E}\left(B \backslash V\left(\partial_{1} \cup \hat{\sigma}_{1}^{\prime} \cup \partial_{2}^{\prime}\right)\right)} A\left(\partial^{\prime},\left(\partial_{1}^{\prime} \cup \partial_{2}^{\prime} ; \omega\right)\right) g_{B \backslash V\left(\partial_{1}\right)}\left(\partial_{1}^{\prime} \cup \partial_{2}^{\prime} \cup \partial^{\prime}\right) Z\left(B \backslash V\left(\partial_{1}\right)\right) \\
& \quad-\sum_{\Gamma \in\left[\Gamma_{0}\right] \partial^{\prime} \in \mathscr{E}^{\prime}\left(B \backslash V\left(\partial_{1} \cup \bar{o}_{1}^{\prime} \cup \hat{\partial}_{2}^{\prime} \cup \Gamma\right)\right)} A\left(\partial^{\prime},\left(\partial_{1}^{\prime} \cup \partial_{2}^{\prime} \cup \Gamma ; \omega\right)\right) \\
& \quad \cdot g_{B \backslash V\left(\partial_{1}\right)}\left(\partial_{1}^{\prime} \cup \partial_{2}^{\prime} \cup \partial^{\prime} \cup \Gamma\right) Z\left(B \backslash V\left(\partial_{1}\right)\right) .
\end{aligned}
$$

One notices that

$$
g_{B \backslash V\left(\hat{c}_{1}\right)}\left(\partial_{1}^{\prime} \cup \partial_{2}^{\prime} \cup \partial^{\prime}\right) Z\left(B \backslash V\left(\partial_{1}\right)\right)=Z\left(B \backslash V\left(\partial_{1} \cup \partial_{1}^{\prime} \cup \partial_{2}^{\prime} \cup \partial^{\prime}\right)\right) \prod_{\Gamma \in \hat{\partial}_{1}^{\prime} \cup \hat{o}_{2}^{\prime} \cup \hat{c}^{\prime}} Z(\operatorname{Int} \Gamma),
$$

by the definition of $g_{\Lambda}(\partial)$ in (3.2.8).

We are now ready to derive an integral equation for $g_{\Lambda}(\partial)$. Let $f$ be a function defined on the set of finite external $q$-contour systems $\mathscr{E}$ in $\mathbb{Z}^{v}$. Such functions form a Banach space $\mathscr{F}_{\xi}$ :

$$
\mathscr{F}_{\xi}=\left\{f: \mathscr{E}\left(\mathbb{Z}^{v}\right) \rightarrow \mathbb{C}:\|f\|=\sup _{\hat{\partial}} \xi^{-|\partial|}|f(\partial)|<\infty\right\},
$$


where $\xi>0$ and $|\partial|=\sum_{\Gamma \in \hat{O}}|\Gamma|$. Let 1 be the function in $\mathscr{F}_{\xi}$ defined by

$$
\mathbf{1}(\phi)=1, \quad \mathbf{1}(\partial)=0 \quad \text { if } \quad \partial \neq \phi .
$$

We introduce the operator $\chi_{\Lambda}$ on $\mathscr{F}_{\xi}$ by

$$
\left(\chi_{\Lambda} f\right)(\partial)=\chi_{\Lambda}(\partial) f(\partial)
$$

where $\chi_{\Lambda}(\partial)=1$ if $\partial \in \mathscr{E}(\Lambda)$ and $\chi_{\Lambda}(\partial)=0$ otherwise.

We next define some operators on $\mathscr{F}_{\xi}$. For a given $\partial \in \mathscr{E}$ and a fixed $\Gamma_{0} \in \partial$, define operators $\mathbf{K}_{2}$ and $\mathbf{K}_{3}$ on $\mathscr{F}_{\xi}$ by

and

$$
\begin{aligned}
& \left(\mathbf{K}_{2} f\right)(\partial)=\sum_{\substack{\hat{o}_{1} \cup \hat{c}_{2}^{\prime} \in \mathscr{E}\left(\operatorname{Int} \\
\hat{o}_{1} \neq \phi\right.}} \sum_{\left.\left.\Gamma_{0}\right): \hat{o}_{1}^{\prime} \in \mathscr{\delta}^{\prime}\left(\mathbb{Z}^{v} \backslash V(\partial)\right)\right)} \int_{\hat{o}_{1}} d \omega \int_{\hat{c}_{2}^{\prime}} d \omega \int_{\hat{\sigma}_{1}^{\prime}} d \omega \\
& \cdot\left[e^{-H\left(\hat{o}_{1}, \omega\right)} / \prod_{\Gamma \in \hat{o}_{1}} Z(\operatorname{Int} \Gamma)\right] \widehat{H}\left(\partial_{1}, \partial_{1}^{\prime} ; \omega\right) G\left(\partial_{1}, \partial_{2}^{\prime} ; \omega\right) \\
& \sum_{\hat{c}^{\prime} \in \mathscr{E}\left(\mathbb{Z}^{v} \backslash V\left(\left(\hat{\imath}-I_{0}\right) \cup\left(\hat{\imath}_{1} \cup \hat{r}_{1}^{\prime} \cup \tilde{r}_{2}^{\prime}\right)\right)\right)} A\left(\partial^{\prime},\left(\partial_{1}^{\prime} \cup \partial_{2}^{\prime} ; \omega\right)\right) \\
& \cdot f\left(\left(\partial-\Gamma_{0}\right) \cup\left(\partial_{1} \cup \partial_{1}^{\prime} \cup \partial_{2}^{\prime} \cup \partial^{\prime}\right)\right),
\end{aligned}
$$

$$
\begin{aligned}
& \left(\mathbf{K}_{3} f\right)(\partial)=-\sum_{\substack{\hat{c}_{1} \cup \hat{\epsilon}_{2}^{\prime} \in \mathscr{E}\left(\operatorname{Int} \Gamma_{0}\right) \\
\hat{c}_{1} \neq \phi}} \sum_{\hat{c}_{1}^{\prime} \in \mathscr{E}\left(\mathbb{Z}^{v} \backslash V(\hat{)})\right)} \int_{\hat{\sigma}_{1}} d \omega \int_{\hat{\sigma}_{2}^{\prime}} d \omega \int_{\hat{c}_{1}^{\prime}} d \omega \\
& \cdot\left[e^{-H\left(\hat{o}_{1} ; \omega\right) /} \prod_{\Gamma \in \hat{o}_{1}} Z(\operatorname{Int} \Gamma)\right] \bar{H}\left(\partial_{1}, \partial_{1}^{\prime} ; \omega\right) G\left(\partial_{1}, \partial_{2}^{\prime} ; \omega\right)
\end{aligned}
$$

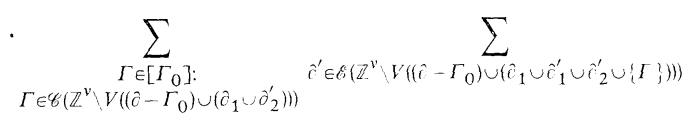

$$
\begin{aligned}
& \text { - } A\left(\partial^{\prime},\left(\partial_{1}^{\prime} \cup \partial_{2}^{\prime} \cup\{\Gamma\} ; \omega\right)\right) f\left(\left(\partial-\Gamma_{0}\right) \cup\left(\partial_{1} \cup \partial_{1}^{\prime} \cup \partial_{2}^{\prime} \cup\{\Gamma\} \cup \partial^{\prime}\right)\right) \text {. }
\end{aligned}
$$

Replacing $B$ by $\Lambda \backslash V\left(\partial-\Gamma_{0}\right)$ in (3.3.12) and (3.3.14), and using (3.3.15) and the definitions in (3.3.18) and (3.3.19), it is not hard to check that

$$
\begin{aligned}
& {\left[\left\{Z(\Lambda \backslash V(\partial)) Z\left(\operatorname{Int} \Gamma_{0}\right)-Z\left((\Lambda \backslash V(\partial)) \cup \operatorname{Int} \Gamma_{0}\right)\right\} \prod_{\Gamma \in \partial-\Gamma_{0}} Z(\operatorname{Int} \Gamma)\right] / Z(\Lambda)} \\
& \quad=\left(\chi_{\Lambda} \mathbf{K}_{2} \chi_{\Lambda} g_{\Lambda}\right)(\partial)+\left(\chi_{\Lambda} \mathbf{K}_{3} \chi_{\Lambda} g_{\Lambda}\right)(\partial),
\end{aligned}
$$

holds for any $\partial \in \mathscr{E}(\Lambda)$ and a fixed $\Gamma_{0} \in \partial$.

We next consider the partition function $Z\left(\Lambda \backslash V\left(\partial-\Gamma_{0}\right)\right)$. Replacing $B$ by $\Lambda \backslash V\left(\partial-\Gamma_{0}\right)$ in (3.3.3) we have

$$
\begin{aligned}
Z & \left(\Lambda \backslash V\left(\partial-\Gamma_{0}\right)\right) \\
& =Z\left((\Lambda \backslash V(\partial)) \cup \operatorname{Int} \Gamma_{0}\right)+\sum_{\Gamma \in\left[\Gamma_{0}\right]} \sum_{\partial^{\prime} \in \mathscr{E}\left(\Lambda \backslash V\left(\hat{c}-\Gamma_{0}\right)\right)} \int_{\Gamma} d \omega \int_{\hat{c}^{\prime}} d \omega e^{-H\left(\Gamma \cup \hat{c}^{\prime} ; \omega\right)} \\
& =Z\left((\Lambda \backslash V(\partial)) \cup \operatorname{Int} \Gamma_{0}\right)+\sum_{\Gamma \in\left[\Gamma_{0}\right]} \int_{\Gamma} d \omega f_{\Lambda \backslash V\left(\hat{c}-\Gamma_{0}\right)}(\{\Gamma\} ; \omega)
\end{aligned}
$$




$$
\begin{aligned}
= & Z\left((\Lambda \backslash V(\partial)) \cup \operatorname{Int} \Gamma_{0}\right)+\sum_{\Gamma \in\left[\Gamma_{0}\right]} \sum_{\hat{c}^{\prime} \in \mathscr{E}\left(\Lambda \backslash V\left(\hat{c}-\Gamma_{0}\right)\right)} \int_{\Gamma} d \omega A\left(\partial^{\prime},(\{\Gamma\} ; \omega)\right) \\
& \cdot g_{\Lambda \backslash V\left(\hat{o}-\Gamma_{0}\right)}\left(\Gamma \cup \partial^{\prime}\right) Z\left(\Lambda \backslash V\left(\partial-\Gamma_{0}\right)\right) .
\end{aligned}
$$

Here we have used (3.2.12) in the last step. We define an operator on $\mathscr{F}_{\xi}$ by

$$
\left(\mathbf{K}_{4} f\right)(\partial)=-\sum_{\Gamma \in\left[\Gamma_{0}\right] \hat{c}^{\prime} \in \mathscr{E}\left(\mathbb{Z}^{\vee} \backslash V\left(\hat{c}-\Gamma_{0}\right)\right)} \int_{\Gamma} d \omega A\left(\partial^{\prime},(\Gamma ; \omega)\right) f\left(\left(\partial-\Gamma_{0}\right) \cup \Gamma \cup \partial^{\prime}\right) .
$$

Then, from (3.2.8), (3.3.21) and the fact that

$$
g_{\Lambda \backslash V\left(\hat{\imath}-\Gamma_{0}\right)}\left(\Gamma \cup \partial^{\prime}\right) Z\left(\Lambda \backslash V\left(\partial-\Gamma_{0}\right)\right)=Z\left(\Lambda \backslash V\left(\partial-\Gamma_{0}\right) \cup \Gamma \cup \partial^{\prime}\right) \prod_{\Gamma^{\prime} \in \Gamma \cup \hat{\imath}^{\prime}} Z\left(\operatorname{Int} \Gamma^{\prime}\right),
$$

if follows that

$$
g_{\Lambda}\left(\partial-\Gamma_{0}\right)=\left[Z\left((\Lambda \backslash V(\partial)) \cup \operatorname{Int} \Gamma_{0}\right) \prod_{\Gamma \in \hat{\Gamma}-\Gamma_{0}} Z(\operatorname{Int} \Gamma)\right] / Z(\Lambda)-\left(\chi_{\Lambda} \mathbf{K}_{4} \chi_{\Lambda} g_{\Lambda}\right)(\partial)
$$

Combining (3.3.20) and (3.3.24) together, we obtain that

Finally, let

$$
g_{\Lambda}\left(\partial-\Gamma_{0}\right)=g_{\Lambda}(\partial)-\left(\chi_{\Lambda}\left(\mathbf{K}_{2}+\mathbf{K}_{3}+\mathbf{K}_{4}\right) \chi_{\Lambda} g_{\Lambda}\right)(\partial) .
$$

$$
\left(\mathbf{K}_{1} f\right)(\partial)=f\left(\partial-\Gamma_{0}\right)
$$

and let

$$
(\mathbf{K} f)(\partial)=\left(\left(\mathbf{K}_{1}+\mathbf{K}_{2}+\mathbf{K}_{3}+\mathbf{K}_{4}\right) f\right)(\partial),
$$

if $\partial \neq \phi$, and $(\mathbf{K} f)(\partial)=0$ otherwise. Since $g_{\Lambda}(\phi)=1$ by the definition in (3.2.8) we obtain the following equation:

$$
g_{\Lambda}=1+\chi_{\Lambda} \mathbf{K} \chi_{\Lambda} g_{\Lambda}
$$

The above relation is the integral equation of a Kirkwood-Salsburg type we were looking for.

\section{Convergence of the Cluster Expansion}

\subsection{Statement of Main Results}

We list our main results on the cluster expansion in this section. For any $\partial \in \mathscr{E}\left(\mathbb{Z}^{v}\right)$, denote

$$
|\partial|=\sum_{\Gamma \in \hat{C}}|\Gamma|, \quad V(\partial)=\bigcup_{\Gamma \in \hat{C}} V(\Gamma), \quad \text { Int } \partial=\bigcup_{\Gamma \in \hat{\partial}} \operatorname{Int} \Gamma .
$$

Let $\delta$ be the metric introduced in Assumption 2.3.2 and for any $\Gamma, \Gamma^{\prime} \in \mathscr{C}$ and $x \in \mathbb{Z}^{v}$, let

$$
\delta(\Gamma, x)=\min \{\delta(y, x): y \in V(\Gamma)\}, \quad \delta\left(\Gamma, \Gamma^{\prime}\right)=\min \{\delta(\Gamma, x): x \in V(\Gamma)\} .
$$

Without loss of generality one may assume that for any $x, y \in \mathbb{Z}^{v}$,

$$
\delta(x, y) \leqq\|x-y\| \text {. }
$$


Otherwise one may replace $\delta(x, y)$ by $\|x-y\|$ in (2.3.5). For any external $q$-contour systems $\partial, \partial^{\prime} \in \mathscr{E}\left(\mathbb{Z}^{v}\right)$, denote

$$
\delta\left(\partial \mid \partial^{\prime}\right)=\max _{x \in V\left(\hat{o}^{\prime}\right)} \min _{\Gamma \in \hat{c}}\{\delta(\Gamma, x)\} .
$$

Note that $\delta\left(\partial \mid \partial^{\prime}\right)$ is not symmetric function on $\mathscr{E} \times \mathscr{E}$.

The following are main results on the cluster expansion:

Theorem 4.1.1. Let a stable $q \in Q$ be chosen. Under Assumption 2.3.2, the following results hold for sufficiently large $\tau$ :

(a) The cluster expansion (3.2.10) is summable absolutely, uniformly in $\Lambda$. Furthermore the bound

$$
\rho_{\Lambda}(\partial) \leqq \exp (-\tau|\partial| / 6)
$$

holds for any $\partial \in \mathscr{E}(\Lambda)$, uniformly in $\Lambda$.

(b) The infinite volume limit

$$
\rho(\partial)=\lim _{\Lambda \rightarrow \mathbb{Z}^{\nu}} \rho_{\Lambda}(\partial)
$$

exists for each $\partial \in \mathscr{E}\left(\mathbb{Z}^{v}\right)$. Furthermore the bound

$$
\left|\rho_{\Lambda}(\partial)-\rho(\partial)\right| \leqq \exp \left\{-\frac{\tau}{6}|\partial|-\delta\left(\partial, \Lambda^{c}\right)\right\}
$$

also holds for each $\partial \in \mathscr{E}\left(\mathbb{Z}^{v}\right)$, where $\delta\left(\partial, \Lambda^{c}\right)=\min \left\{\delta(x, y): x \in \operatorname{supp} \partial, y \in \Lambda^{c}\right\}$.

Theorem 4.1.2. Under the assumptions the same as those in Theorem 4.1.1, the cluster property holds: For any $\partial, \partial^{\prime} \in \mathscr{E}\left(\mathbb{Z}^{v}\right)$ with $\partial \cup \partial^{\prime} \in \mathscr{E}\left(\mathbb{Z}^{v}\right)$,

$$
\rho\left(\partial \cup \partial^{\prime}\right)-\rho(\partial) \rho\left(\partial^{\prime}\right) \rightarrow 0,
$$

as $\operatorname{dist}\left(V(\partial), V\left(\partial^{\prime}\right)\right)$ tends to infinity.

The main results (Theorem 2.3.4(a) and Theorem 2.3.4(b)) in Sect. 2.3. are just consequences of the above results. The above results will be proved by using the following results:

Proposition 4.1.3. Under the conditions as in Theorem 4.1.1, the infinite volume limit

$$
g(\partial)=\lim _{\Lambda \rightarrow \mathbb{Z}^{v}} g_{\Lambda}(\partial)
$$

exist for any $\partial \in \mathscr{E}\left(\mathbb{Z}^{v}\right)$. The integral equation

$$
g=\mathbf{1}+\mathbf{K} g,
$$

holds on the Banach space $\mathscr{F}_{\xi}, \xi=\exp (+\tau / 8)$.

Proposition 4.1.4. Under the conditions as in Theorem 4.1.1, the bound

$$
\left|g_{\Lambda}(\partial)-g(\partial)\right| \leqq \exp \left(\frac{\tau}{8}|\partial|-\delta\left(\partial, \Lambda^{c}\right)\right),
$$

holds for any finite $\Lambda \subset \mathbb{Z}^{v}$ and any $\partial \in \mathscr{E}(\Lambda)$, where $\delta\left(\partial, \Lambda^{c}\right)$ is the distance between supp $\partial$ and $\Lambda^{c}$ with respect to the metric $\delta$. 
Proposition 4.1 .4 will be also used to investigate the structure of phase diagram in the sequel [13]. In the rest of the paper we will produce the proofs of our results.

\subsection{Some Useful Estimates}

In this section, we derive useful estimates which will be used to prove the main results. We first state a Peierls type estimate from the reference [18].

Lemma 4.2.1. [Lemma 2.7 of [18]]. Let $a=\max \left\{v \ln (2 s+1), \ln |\Omega|+3^{v}\right\}$, where $s \geqq 1$ is the parameter used to define contours. Then

$$
\left|\left\{\Gamma \in \mathscr{C}\left(\mathbb{Z}^{v}\right): d(x, V(\Gamma)) \leqq 1, \quad|\Gamma|=n\right\}\right| \leqq e^{a n}
$$

holds for each $x \in \mathbb{Z}^{v}$.

In the rest of this paper we assume that Assumption 2.3.2 holds and that a stable ground state $q \in Q$ is chosen. The results in the following proposition are consequences of the regularity condition (Assumption 2.3.2).

Proposition 4.2.2. (a) Let $B \subset \mathbb{Z}^{v}$ be a bounded region and let $\partial, \partial^{\prime} \in \mathscr{E}$ such that $V(\partial) \subset B$ and $V\left(\partial^{\prime}\right) \subset B^{c}$. Then for any configuration $\omega$ for which $\partial \cup \partial^{\prime}$ is the external q-boundary of $\omega$, the bound

$$
\sum_{\Gamma \in \hat{o}} \sum_{\Gamma^{\prime} \in \hat{O}^{\prime}} e^{\delta\left(\Gamma, \Gamma^{\prime}\right)}\left|\Phi_{2}\left(\Gamma, \Gamma^{\prime} ; \omega\right)\right| \leqq \min \{\tau|\mathrm{bd}(V(\partial))| / 48, \tau|\operatorname{bd}(B)| / 48\},
$$

holds uniformly in $\omega$, where $\mathrm{bd}(B)$ is the boundary of $B$.

(b) For any $q$-contour $\Gamma$, the inequality

$$
\sum_{\Gamma^{\prime} \in \mathscr{Q}_{q}\left(\mathbb{Z}^{v} \backslash V(\Gamma)\right)} \sup _{\omega}\left|\Phi_{2}\left(\Gamma, \Gamma^{\prime} ; \omega\right)\right| \exp \left(\delta\left(\Gamma, \Gamma^{\prime}\right)-\tau\left|\Gamma^{\prime}\right| / 48\right) \leqq \tau|\operatorname{bd}(V(\Gamma))| / 48,
$$

holds for sufficiently large $\tau$

(c) Let $B$ be a finite region in $\mathbb{Z}^{v}$. Then for any given $\partial \in \mathscr{E}\left(\mathbb{Z}^{v} \backslash B\right)$ the inequality

$$
\sum_{\Gamma \in \mathscr{C}(B)} \sum_{\Gamma^{\prime} \in \hat{c}} \sup _{\omega}\left|\Phi_{2}\left(\Gamma, \Gamma^{\prime} ; \omega\right)\right| \exp \left(\delta\left(\Gamma, \Gamma^{\prime}\right)-\tau|\Gamma| / 48\right) \leqq \tau|\operatorname{bd}(B)| / 48,
$$

holds for sufficiently large $\tau$.

(d) For any finite region $B$ in $\mathbb{Z}^{v}$, the inequality

$$
\sum_{\Gamma \in \mathscr{C}(B)} \sum_{\Gamma^{\prime} \in \mathscr{C}\left(\mathbb{Z}^{\vee} \backslash B\right)} \sup _{\omega}\left|\Phi_{2}\left(\Gamma, \Gamma^{\prime} ; \omega\right)\right| \exp \left(\delta\left(\Gamma, \Gamma^{\prime}\right)-\tau\left|\Gamma \cup \Gamma^{\prime}\right| / 48\right) \leqq \tau|\mathrm{bd}(B)| / 48,
$$

holds for sufficiently large $\tau$.

Since the method of the proof of the above proposition is somewhat lengthy and is not used in other places, we postponed the proof to the appendix at the end of this paper.

We next consider the contour functional $g_{\Lambda}(\partial)$ defined in (3.2.8). We have the following uniform estimate:

Lemma 4.2.3. The bound

$$
\left|g_{\Lambda}(\partial)\right| \leqq \exp (\tau|\partial| / 24)
$$

holds for any $\partial \in \mathscr{E}(\Lambda)$, uniformly in $\Lambda$. 
Proof. For any given $\partial \in \mathscr{E}(\Lambda)$ it follows from (3.1.4) that

$$
Z(\Lambda) \geqq \sum_{\substack{\hat{\sigma}^{\prime} \in \mathscr{E}(\Lambda \backslash V(\partial)) \\ \hat{\partial}^{\prime \prime} \in \mathscr{E}^{\prime}(\operatorname{Int} \hat{\partial})}} \int_{\hat{o}^{\prime}} d \omega \int_{\hat{o}^{\prime \prime}} d \omega e^{-H\left(\hat{\sigma}^{\prime} \cup \hat{o}^{\prime \prime} ; \omega\right)} .
$$

Writing $H\left(\partial^{\prime} \cup \partial^{\prime \prime} ; \omega\right)=H\left(\partial^{\prime} ; \omega\right)+H\left(\partial^{\prime \prime} ; \omega\right)+\Phi_{2}\left(\partial^{\prime}, \partial^{\prime \prime} ; \omega\right)$, using (4.2.1) and the fact that $|\mathrm{bd}(V(\partial))| \leqq|\partial|$, we have

$$
Z(\Lambda) \geqq Z(\Lambda \backslash V(\partial)) \exp (-\tau|\partial| / 48) \sum_{\hat{\partial}^{\prime \prime} \in \mathscr{E}^{\circ}(\operatorname{Int} \hat{\partial})} \int_{\hat{\partial}^{\prime \prime}} d \omega e^{-H\left(\hat{\partial}^{\prime \prime} ; \omega\right)} .
$$

Let $\partial=\left\{\Gamma_{1}, \Gamma_{2}, \ldots, \Gamma_{n}\right\}$ and let $\partial^{\prime \prime}=\left\{\partial_{1}, \partial_{2}, \ldots, \partial_{n}\right\}$, where $\partial_{i} \in \mathscr{E}\left(\operatorname{Int} \Gamma_{i}\right)$. Write

$$
H\left(\partial^{\prime \prime} ; \omega\right)=\sum_{i=1}^{n} H\left(\partial_{i} ; \omega\right)+\sum_{i \neq j=1}^{n} \Phi_{2}\left(\partial_{i}, \partial_{j}: \omega\right) \text {. }
$$

We use (4.2.1) again (with $B=V(\partial)$ ) to conclude that

$$
Z(\operatorname{Int} \partial)=\sum_{\hat{c}^{\prime \prime} \in \delta^{\prime}(\operatorname{Int} \hat{c})} \int_{\hat{c}^{\prime \prime}} d \omega e^{-H\left(\hat{c}^{\prime \prime} ; \omega\right)} \geqq\left(\prod_{\Gamma \in \hat{o}} Z(\operatorname{Int} \Gamma)\right) \exp (-\tau|\partial| / 48) \text {. }
$$

The lemma follows from (3.2.8), (4.2.5) and (4.2.6).

Recall the definitions of $\tilde{K}\left(\partial, \partial^{\prime} ; \omega\right)$ and $\delta\left(\partial \mid \partial^{\prime}\right)$ given in (3.2.7) and (4.1.4) respectively.

Lemma 4.2.4. For any $\partial \in \mathscr{E}$ and for sufficiently large $\tau$, the bound

$$
\sum_{\phi \neq \partial^{\prime} \in \tilde{\sigma}^{\prime}\left(\mathbb{Z}^{v} \backslash V(\hat{\partial})\right)} \int_{\partial} d \omega \sup _{\omega \in \Omega\left(\partial^{\prime}\right)}\left|\tilde{K}\left(\partial, \partial^{\prime} ; \omega\right)\right| \exp \left(\delta\left(\partial \mid \partial^{\prime}\right)-\tau\left|\partial^{\prime}\right| / 12\right) \leqq e^{-\tau|c| / 4} .
$$

holds, where $\Omega\left(\partial^{\prime}\right)=\underset{\Gamma^{\prime} \in \hat{\sigma}^{\prime}}{\times} \Omega\left(\Gamma^{\prime}\right)$,

Proof. Since $\max _{x \in V\left(\hat{\sigma}^{\prime}\right)}\left\{\delta(\partial, x) \leqq \max _{\Gamma^{\prime} \in \hat{o}^{\prime}}\left\{\delta\left(\partial, \Gamma^{\prime}\right)+\operatorname{diam}\left(\Gamma^{\prime}\right)\right\}\right.$

$$
\delta\left(\partial \mid \partial^{\prime}\right) \leqq \sum_{\Gamma^{\prime} \in \hat{o}^{\prime}}\left\{\delta\left(\partial, \Gamma^{\prime}\right)+\left|\Gamma^{\prime}\right|\right\} .
$$

From the definition of $\tilde{K}\left(\partial, \partial^{\prime} ; \omega\right)$ and (4.2.7) it follows that for $\tau / 24>1$ the expression in Lemma 4.2 .4 is bounded by

$$
\sum_{\phi \neq \hat{\delta}^{\prime} \in \delta^{\prime}(\Lambda \backslash V(\hat{\sigma}))} \int_{\hat{\delta}} d \omega K(\partial ; \omega) \sup _{\omega \in \Omega\left(\hat{\sigma}^{\prime}\right)} \prod_{\Gamma^{\prime} \in \partial^{\prime}}\left\{\left|g\left(\partial, \Gamma^{\prime} ; \omega\right)\right| e^{\delta\left(\hat{\partial}, \Gamma^{\prime}\right)-\tau \mid \Gamma^{\prime} / 24}\right\} .
$$

A direct calculation yields

$$
\begin{aligned}
& \sum_{\phi \neq \hat{\partial}^{\prime} \in \mathscr{E}(\Lambda \backslash V(\hat{\partial}))} \prod_{\Gamma^{\prime} \in \hat{o}^{\prime}}\left\{\left|g\left(\partial, \Gamma^{\prime} ; \omega\right)\right| e^{\delta\left(\hat{\sigma}, \Gamma^{\prime}\right)-\tau \mid \Gamma^{\prime} / 24}\right\} \\
& =\sum_{n=1}^{\infty} \sum_{\left\{\Gamma_{1}, \ldots, \Gamma_{n}\right\} \in \mathscr{E}\left(\mathbb{Z}^{v} \backslash V(\hat{\partial})\right)} \prod_{i=1}^{n}\left|g\left(\partial, \Gamma_{i} ; \omega\right)\right| e^{\delta\left(\hat{o}, \Gamma_{i}\right)-\tau\left|\Gamma_{l}\right| / 24} \\
& \leqq \sum_{n=1}^{\infty} \frac{1}{n !} \prod_{i=1}^{n}\left(\sum_{\Gamma_{i} \in \mathscr{C}\left(\mathbb{Z}^{v} \backslash V(\partial)\right)}\left|g\left(\partial, \Gamma_{i} ; \omega\right)\right| e^{\delta\left(\hat{o}, \Gamma_{i}\right)-\tau\left|\Gamma_{i}\right| / 24}\right) \\
& \leqq \exp \left\{\sum_{\Gamma \in \mathscr{G}\left(\mathbb{Z}^{v} \backslash V(\partial)\right)}\left|g\left(\partial, \Gamma^{\prime} ; \omega\right)\right| e^{\delta(\hat{\partial}, \Gamma)-\tau|\tau| / 24}\right\}-1 .
\end{aligned}
$$


We note that

$$
|g(\partial, \Gamma ; \omega)|=\left|\Phi_{2}(\partial, \Gamma ; \omega) \int_{0}^{1} d s e^{-s \Phi_{2}(\hat{\theta}, \Gamma \cdot \omega)}\right| \leqq\left|\Phi_{2}(\partial, \Gamma ; \omega)\right| e^{\tau|\Gamma| / 48} .
$$

Here we have used the fact that

$$
\sup _{\omega}\left|\Phi_{2}(\partial, \Gamma ; \omega)\right| \leqq \sum_{\Gamma^{\prime} \in \partial} \sup _{\omega}\left|\Phi_{2}\left(\Gamma^{\prime}, \Gamma ; \omega\right)\right| \leqq \tau|\Gamma| / 48
$$

by (4.2.1). Using (4.2.10) and (4.2.2) we obtain that for sufficiently large $\tau$

$$
\begin{aligned}
\sum_{\Gamma \in \mathscr{G}\left(\mathbb{Z}^{v} \backslash V(\hat{\partial})\right)}|g(\partial, \Gamma ; \omega)| e^{\partial(\hat{c}, \Gamma)-\tau|\Gamma| / 24} & \leqq \sum_{\Gamma \in \mathscr{G}\left(\mathbb{Z}^{v} \backslash V(\hat{\partial})\right)}\left|\Phi_{2}(\partial, \Gamma ; \omega)\right| e^{\partial(\hat{c}, \Gamma)-\tau|\Gamma| / 48} \\
& \leqq \sum_{\Gamma \in \mathscr{G}\left(\mathbb{Z}^{v} \backslash V(\hat{\partial})\right)} \sum_{\Gamma^{\prime} \in \hat{i}}\left|\Phi\left(\Gamma, \Gamma^{\prime} ; \omega\right)\right| e^{\delta\left(\Gamma^{\prime}, \Gamma\right)-\tau|\Gamma| / 48} \\
& \leqq \tau|\operatorname{bd}(V(\partial))| / 48
\end{aligned}
$$

Thus we conclude that

$$
(4.2 .9) \leqq e^{\tau|\vec{c}| / 48}
$$

On the other hand, we use (2.2.9), (4.2.1) and the stability condition in Definition 2.3.3 to obtain the bound

$$
\int_{\hat{c}} d \omega K(\partial ; \omega)=\int_{\hat{\partial}} d \omega e^{-H(\hat{c} ;(\omega)} / \prod_{\Gamma \in \partial} Z(\operatorname{Int} \Gamma) \leqq \exp (-15 \tau|\partial| / 48)
$$

The lemma follows from (4.2.8), (4.2.9), (4.2.12) and (4.2.13).

We produce estimates which will be used in the proofs of the main result.

Proposition 4.2.5. (a) Let $B \subset \mathbb{Z}^{v}$ be finite and let $\bar{H}\left(\partial_{1}, \partial_{1}^{\prime} ; \omega\right)$ be defined as in (3.3.6). Then for sufficiently large $\tau$, the bound

$$
\sum_{\hat{o}_{1} \in \mathscr{E}(B)} \sum_{\hat{\sigma}_{1}^{\prime} \in \mathscr{E}\left(\mathbb{Z}^{v} \backslash B\right)} \sup _{\omega}\left|\bar{H}\left(\partial_{1}, \partial_{1}^{\prime} ; \omega\right)\right| e^{\delta\left(\hat{c}_{1} \mid \hat{\sigma}_{1}^{\prime}\right)-\tau\left|\hat{o}_{1} \cup \hat{\sigma}_{1}^{\prime}\right| / 8} \leqq \exp (\tau|\operatorname{bd}(B)| / 12)
$$

holds.

(b) For $\partial \in \mathscr{E}\left(\mathbb{Z}^{v}\right)$ and $\partial^{\prime} \in \mathscr{E}\left(\mathbb{Z}^{v} \backslash V(\partial)\right)$, let $A\left(\partial^{\prime} ;(\partial ; \omega)\right)$ be defined as in (3.2.11). Then for sufficiently large $\tau$, the bound,

holds.

$$
\sum_{\phi \neq \hat{\sigma}^{\prime} \in \mathscr{\delta}\left(\mathbb{Z}^{v} \backslash V(\hat{c})\right) \hat{e}} \int_{d} d \omega\left|A\left(\partial^{\prime} ;(\partial ; \omega)\right)\right| e^{\delta\left(\hat{\theta} \mid \hat{o}^{\prime}\right)+\tau\left|\hat{\sigma}^{\prime}\right| / 8} \leqq e^{-\tau|\hat{\theta}| / 4}
$$

Proof. (a) From the definition of $\bar{H}\left(\partial_{1}, \partial_{1}^{\prime} ; \omega\right)$ in (3.3.6) and from (4.2.7) it follows that for $\tau / 24>1$,

$$
\begin{aligned}
& \sum_{\substack{\phi \neq \hat{c}_{1} \in \mathscr{E}^{\prime}(B) \\
\phi \neq \hat{\sigma}_{1}^{\prime} \in \bar{E}^{G}\left(\mathbb{Z}^{V} \backslash B\right)}}\left|\bar{H}\left(\partial, \partial_{1}^{\prime} ; \omega\right)\right| e^{\delta\left(\hat{\sigma}_{1} \mid \hat{\sigma}_{1}^{\prime}\right)-\tau\left|\hat{\sigma}_{1} \cup \hat{c}_{1}^{\prime}\right| / 8} \\
& \leqq \sum_{n=1}^{\infty} \sum_{\left\{\Gamma_{1}, \Gamma_{2}, \ldots, \Gamma_{n}\right\} \in \mathscr{E}(B)} \sum_{\substack{\left\{\hat{\sigma}_{1}, \ldots, \hat{\sigma}_{n}\right\} \\
\cup \hat{\sigma}_{1} \in \mathscr{E}\left(\mathbb{Z}^{N} \backslash B\right), \hat{\sigma}_{i} \neq \phi}} \\
& \cdot\left(\prod_{i=1}^{n}\left(\prod_{I \in \hat{c}_{i}}\left|g\left(\Gamma, \Gamma_{i} ; \omega\right)\right| e^{\delta\left(\Gamma, \Gamma_{t}\right)}\right) e^{-\tau\left|\Gamma_{i}\right| / 8}\right) e^{-\tau\left|\hat{c}_{i} \in \dot{E}\left(\mathbb{Z}^{\nu} \backslash B\right) \hat{c}_{i}\right| / 12}
\end{aligned}
$$




$$
\begin{aligned}
\leqq & \sum_{n=1}^{\infty} \frac{1}{n !}\left\{\sum_{\Gamma_{1} \in \mathscr{G}(B)} \cdots \sum_{\Gamma_{n} \in \mathscr{C}(B)} \sum_{\substack{\left\{\hat{c}_{i}, \ldots, \hat{c}_{n}\right\} \\
\cup \hat{o}_{i} \in \mathscr{E}\left(\mathbb{Z}^{\top} \backslash B\right)}}\right. \\
& \left.\cdot\left(\prod_{i=1}^{n}\left[\prod_{\Gamma \in \hat{c}_{i}}\left|g\left(\Gamma, \Gamma_{i} ; \omega\right)\right| e^{\delta\left(\Gamma, \Gamma_{i}\right)}\right] e^{-\tau\left|\Gamma_{i}\right| / 8}\right) e^{-\tau\left|\bigcup_{i=1}^{n} \hat{o}_{i}\right| / 12}\right\} .
\end{aligned}
$$

In order to prove the proposition we use a finite interaction method. As the first step we define

$$
A_{n}\left(\Gamma_{n}\right) \equiv \sum_{\phi \neq \hat{c}_{n} \in \mathscr{\delta}\left(\mathbb{Z}^{v} \backslash B\right)}\left(\prod_{I \in \hat{c}_{n}}\left|g\left(\Gamma, \Gamma_{n}\right)\right| e^{\delta\left(\Gamma, \Gamma_{n}\right)}\right) e^{-\left|\Gamma_{n}\right| 8} e^{-\tau\left|\bigcup_{i=1}^{n} \hat{c}_{i} \backslash \bigcup_{i=1}^{n-1} \hat{c}_{i}\right| / 12} .
$$

We write $\partial_{n}=\partial_{n}^{\prime} \cup \partial_{n}^{\prime \prime}$, where $\hat{\partial}_{n}^{\prime} \subset \bigcup_{i=1}^{n-1} \partial_{i}$, and $\partial_{n}^{\prime \prime} \in \mathscr{E}\left(\mathbb{Z}^{v} \backslash\left(B \cup\left(V\left(\bigcup_{i=1}^{n-1} \partial_{i}\right)\right)\right)\right)$. Let

$$
\begin{aligned}
& A_{n}^{\prime}\left(\Gamma_{n}\right) \equiv \sum_{\substack{n-1 \\
\phi \neq \hat{\delta}_{n}^{\prime} \subset \bigcup_{i=1}^{n-1} \hat{c}_{i}}}\left(\prod_{\Gamma \in \hat{\tau}_{n}^{\prime}}\left|g\left(\Gamma, \Gamma_{n} ; \omega\right)\right| e^{\delta\left(\Gamma, \Gamma_{n}\right)}\right) e^{-\tau\left|\Gamma_{n}\right| / 8} . \\
& A_{n}^{\prime \prime}\left(\Gamma_{n}\right) \equiv \sum_{\phi \neq \hat{c}_{n}^{\prime \prime}} \sum_{\substack{n-1 \\
\hat{c}_{n}^{\prime} \subset \bigcup \bigcup_{i=1}}}\left(\prod_{\Gamma \in \hat{o}_{i}^{\prime} \cup \hat{c}_{n}^{\prime \prime}}\left|g\left(\Gamma, \Gamma_{n} ; \omega\right)\right| e^{\delta\left(\Gamma, \Gamma_{n}\right)}\right) \\
& \cdot e^{-\tau\left|\Gamma_{n}\right| / 8} \prod_{\Gamma \in \hat{c}_{n}^{\prime \prime}} e^{-\tau|\Gamma| / 12}
\end{aligned}
$$

We decompose the sum over $\partial_{n}$ in $(4.2 .15)$ by

$$
\sum_{\phi \neq \hat{c}_{n}}=\sum_{\phi \neq \hat{c}_{n}^{\prime} \cup \hat{c}_{n}^{\prime \prime}}=\sum_{\substack{\phi \neq \hat{c}_{n}^{\prime} \\ \hat{c}_{n}^{\prime \prime}=\phi}}+\sum_{\phi \neq \hat{c}_{n}^{\prime \prime}} \sum_{\hat{\kappa}_{n}^{\prime}} \text {. }
$$

They, by the definition of $A_{n}\left(\Gamma_{n}\right)$ in $(4.2 .15)$ we have

$$
A_{n}\left(\Gamma_{n}\right)=A_{n}^{\prime}\left(\Gamma_{n}\right)+A_{n}^{\prime \prime}\left(\Gamma_{n}\right) .
$$

We will calculate $A_{n}^{\prime}\left(\Gamma_{n}\right)$ and $A_{n}^{\prime \prime}\left(\Gamma_{n}\right)$.

Let us first consider $A_{n}^{\prime}\left(\Gamma_{n}\right)$. Using the relation (4.2.10) for $\partial=\{\Gamma\}$ and (4.2.1) we obtain that

$$
\prod_{\Gamma \in \hat{o}_{n}^{\prime}}\left|g\left(\Gamma, \Gamma_{n} ; \omega\right)\right| e^{\delta\left(\Gamma, \Gamma_{n}\right)} \leqq\left(\prod_{\Gamma \in \hat{o}_{n}^{\prime}}\left|\Phi_{2}\left(\Gamma, \Gamma_{n} ; \omega\right)\right| e^{\delta\left(\Gamma \cdot \Gamma_{n}\right)}\right) e^{\tau\left|\Gamma_{n}\right| / 48}
$$

We use the method employed in (4.2.9) and the above bound to obtain

$$
\begin{aligned}
A_{n}^{\prime}\left(\Gamma_{n}\right) & \leqq\left(\exp \left\{\sum_{\substack{n-1 \\
\Gamma \in \bigcup_{i=1} \hat{c}_{i}}}\left|\Phi_{2}\left(\Gamma, \Gamma_{n} ; \omega\right)\right| e^{\delta\left(\Gamma, \Gamma_{n}\right)}\right\}-1\right) e^{-5 \tau\left|\Gamma_{n}\right| / 48} \\
& \leqq\left(\sum_{\substack{n-1 \\
\Gamma \in \bigcup_{i=1} \hat{c}_{i}}}\left|\Phi_{2}\left(\Gamma, \Gamma_{n} ; \omega\right)\right| e^{\delta\left(\Gamma, \Gamma_{n}\right)}\right) e^{-\tau\left|\Gamma_{n}\right| / 12} .
\end{aligned}
$$


Here we have used the first relation in (4.2.10), and (4.2.1) to get the second inequality. And so (4.2.3) implies that

$$
A_{n}^{\prime} \equiv \sum_{\Gamma_{n} \in \mathscr{C}(B)} A_{n}^{\prime}\left(\Gamma_{n}\right) \leqq \tau|\mathrm{bd}(B)| / 48
$$

On the other hand, observe that by $(4.2 .20)$

$$
\begin{aligned}
\sum_{\substack{\hat{c}_{n}^{\prime} \subset-1 \\
\hat{c}_{i=1} \bigcup_{i} \prod_{i \in \hat{o}_{n}^{\prime}}}}\left|g\left(\Gamma, \Gamma_{n}\right)\right| e^{\delta\left(\Gamma, \Gamma_{n}\right)} & \leqq \exp \left(\sum_{\substack{n-1 \\
\Gamma \in \bigcup_{i=1} \hat{o}_{i}}}\left|\Phi_{2}\left(\Gamma, \Gamma_{n} ; \omega\right)\right| e^{\delta\left(\Gamma, \Gamma_{n}\right)}\right) e^{\tau\left|\Gamma_{n}\right| / 48} \\
& \leqq e^{\tau\left|\Gamma_{n}\right| / 24}
\end{aligned}
$$

Here we have used (4.2.1) to get the last inequality. Using the above bound into (4.2.17) we obtain

$$
A_{n}^{\prime \prime}\left(\Gamma_{n}\right) \leqq \sum_{\phi \neq i^{\prime \prime} \in \delta^{\mathscr{E}}\left(\mathbb{Z}^{\nu} \backslash B\right)}\left(\prod_{\Gamma \in \hat{C}^{\prime \prime}}\left|g\left(\Gamma, \Gamma_{n} ; \omega\right)\right| e^{\delta\left(\Gamma, \Gamma_{n}\right)-\tau|\Gamma| / 12}\right) e^{-\tau\left|\Gamma_{n}\right| / 12} .
$$

Writing $\partial_{n}^{\prime \prime}=\left\{\Gamma_{1}^{\prime \prime}, \Gamma_{2}^{\prime \prime}, \ldots, \Gamma_{n}^{\prime \prime}\right\}$, using (4.2.20) and (4.2.2) we obtain

$$
\begin{aligned}
A_{n}^{\prime \prime}\left(\Gamma_{n}\right) & \leqq \sum_{m=1}^{\infty} \frac{1}{m !}\left\{\prod_{j=1}^{m}\left(\sum_{\Gamma_{j}^{\prime \prime} \in \mathscr{E}\left(B^{c}\right)}\left|g\left(\Gamma_{j}^{\prime \prime}, \Gamma_{n} ; \omega\right)\right| e^{\delta\left(\Gamma_{j}^{\prime \prime}, \Gamma_{n}\right)-\tau\left|\Gamma_{j}^{\prime \prime}\right| / 12}\right)\right\} e^{-\tau\left|\Gamma_{n}\right| / 12} \\
& \leqq\left(\exp \left\{\sum_{\Gamma \in \mathscr{G}\left(B^{c}\right)}\left|\Phi_{2}\left(\Gamma, \Gamma_{n} ; \omega\right)\right| e^{\delta\left(\Gamma, \Gamma_{n}\right)-\tau|\Gamma| / 18}\right\}-1\right) e^{-\tau \mid \Gamma_{n} / 18} \\
& \leqq\left\{\sum_{\Gamma \in \mathscr{C}\left(B^{c}\right)} \mid \Phi_{2}\left(\Gamma, \Gamma_{n} ; \omega\right) e^{\delta\left(\Gamma, \Gamma_{n}\right)-\tau|\Gamma| / 24}\right\} e^{-\tau_{i}\left|\Gamma_{n}\right| / 18},
\end{aligned}
$$

and so by (4.2.4) and above inequality we obtain

$$
A_{n}^{\prime \prime} \equiv \sum_{\Gamma_{n} \varepsilon \mathscr{G}(B)} A_{n}^{\prime \prime}\left(\Gamma_{n}\right) \leqq \tau|\mathrm{bd}(B)| / 48
$$

Combining (4.2.19), (4.2.21) and (4.2.23) we conclude that

$$
A_{n} \equiv \sum_{\Gamma \in \mathscr{C}(B)} A_{n}\left(\Gamma_{n}\right) \leqq \tau|\mathrm{bd}(B)| / 24 .
$$

Using the above procedure $n$-times in (4.2.14) we prove that the expression in (4.2.14) is bounded by

$$
\sum_{n=1}^{\infty} \frac{1}{n !}(\tau|\operatorname{bd}(B)| / 24)^{n}=e^{\tau \mid \mathrm{bd}(B) / 24}-1 .
$$

This proves the part (a) of the proposition completely.

(b) From the definition of $A\left(\partial^{\prime},(\partial ; \omega)\right)$ in (3.2.11), and from the fact that $\delta\left(\partial \mid \bigcup_{i=1}^{n} \partial_{i}\right) \leqq \delta\left(\partial \mid \partial_{1}\right)+\delta\left(\partial_{1} \mid \partial_{2}\right)+\cdots+\delta\left(\partial_{n-1} \mid \partial_{n}\right)$ it follows that

$$
\sum_{\phi \neq \hat{\sigma}^{\prime} \in \delta(\mathbb{Z} \backslash \backslash V(\hat{c}))} \int_{\hat{c}} d \omega\left|A\left(\partial^{\prime},(\partial ; \omega)\right)\right| e^{\delta\left(\hat{\sigma} \hat{o}^{\prime}\right)+\tau\left|\hat{\sigma}^{\prime}\right| / 8}
$$




$$
\begin{aligned}
\leqq & \sum_{n=1}^{\infty} \sum_{\partial_{1}} \cdots \sum_{\partial_{n}} \int_{\partial} d \omega \prod_{i=1}^{n} \int_{\hat{o}_{i}} d \omega\left|K\left(\partial, \partial_{1}, \ldots, \partial_{n} ; \omega\right)\right| \\
& \cdot e^{\delta\left(\hat{\partial} \mid \hat{c}_{1}\right)}\left(\prod_{i=1}^{n} e^{\delta\left(\hat{o}_{1-1} \mid \hat{o}_{i}\right)+\tau\left|\hat{o}_{i}\right| / 18}\right) .
\end{aligned}
$$

The inequality (4.2.13), Lemma 4.2 .4 and the fact that $|\Gamma| \geqq 4$ for any $\Gamma \in \mathscr{C}$ yield

$$
\begin{aligned}
& \sum_{\phi \neq \hat{o}_{n}} \int_{\partial_{n}} d \omega\left|\tilde{K}\left(\partial_{n-1}, \partial_{n} ; \omega\right) K\left(\partial_{n} ; \omega\right)\right| e^{\delta\left(\hat{o}_{n-1} \mid \hat{o}_{n}\right)+\tau\left|\hat{o}_{n}\right| / 8} \\
& \quad \leqq \sum_{\phi \neq \hat{o}_{n} \omega \in \Omega\left(\hat{o}_{n}\right)} \sup \left|\tilde{K}\left(\partial_{n-1} \partial_{n} ; \omega\right)\right| e^{\delta\left(\hat{c}_{n-1} \mid \hat{o}_{n}\right)-7 \tau\left|\hat{o}_{n}\right| / 48} \\
& \quad \leqq e^{-\tau\left|\hat{o}_{n-1}\right| / 4} \cdot e^{-\tau / 12} .
\end{aligned}
$$

Using the above procedure (and Lemma 4.2.4) n-times and the definition $K\left(\partial, \ldots, \partial_{n} ; \omega\right)$ in $(3.2 .7)$ we conclude that $(4.2 .24)$ is bounded by

$$
\sum_{\hat{\sigma}_{1}} \int_{\partial} d \omega \int_{\hat{o}_{1}} d \omega\left|\tilde{K}\left(\partial, \partial_{1} ; \omega\right)\right| e^{\delta\left(\hat{\sigma} \mid \hat{c}_{i}\right)-\tau\left|\hat{c}_{1}\right| / 8}\left(1-e^{-\tau / 12}\right)^{-1} \leqq e^{-\tau|\hat{c}| / 4}
$$

This proves the part (b) of Proposition 4.2 .5 completely.

\subsection{Proof of Convergence}

In this section we prove Theorem 4.1.1, Proposition 4.1.3 and Proposition 4.1.4. The Proof of the cluster property is postponed to the next section.

Proof of Theorem 4.1.1 (under the assumption that Proposition 4.1.3 and Proposition 4.1 .4 hold).

(a) From (3.2.10), (4.2.13), Lemma 4.2.3 and Proposition 4.2.5(b) it follows that

$$
\rho_{\Lambda}(\partial) \leqq K(\partial) e^{\tau|\hat{\sigma}| / 24}+\sum_{\phi \neq \hat{o}^{\prime} \in \delta^{\prime}(\Lambda \backslash V(\hat{\partial}))} \int_{\hat{\sigma}} d \omega\left|A\left(\partial^{\prime} ;(\hat{\partial} ; \omega)\right)\right| e^{\tau\left|\hat{\sigma} \cup \hat{o}^{\prime}\right| / 24} \leqq e^{-\tau|\hat{\partial}| / \hat{\sigma}},
$$

for sufficiently large $\tau$. This proves part (a).

(b) By part (a) of the theorem, the cluster expansion (3.2.14) is summable absolutely and uniformly in $\Lambda$. Thus, the existence of the limit follows from (3.2.14) and Proposition 4.1.3. Notice that $-\delta\left(\partial \mid \partial^{\prime}\right)-\delta\left(\partial \cup \partial^{\prime}, \Lambda^{c}\right) \leqq-\delta\left(\partial, \Lambda^{c}\right)$, where $\delta\left(\partial \mid \partial^{\prime}\right)$ is defined in (4.1.4). Since $\left|\rho_{\Lambda}(\partial)-\rho(\partial)\right| \leqq \sum_{\hat{c}^{\prime}} \int_{\hat{c}} d \omega\left|A\left(\hat{\partial}^{\prime},(\partial ; \omega)\right)\right| \mid\left(g_{\Lambda}\left(\partial \cup \partial^{\prime}\right)\right)-$ $g\left(\partial \cup \partial^{\prime}\right) \mid$, the bound follows from Proposition 4.1.4 and Proposition 4.2.5(b).

In order to show Proposition 4.1.3 and Proposition 4.1.4 we will use a modification of the method used in [18]. For each finite subset $W \subset \mathbb{Z}^{v}$ we define the norm $\|f\|_{W}$ of external boundary functionals $f$ defined on $\mathscr{E}\left(\mathbb{Z}^{v}\right)$ by

$$
\|f\|_{W}=\sup _{\partial \in \mathscr{E}}|f(\partial)| \exp \left(\delta\left(\partial, W^{c}\right)-\tau|\partial| / 8\right)
$$

where

$$
\delta\left(\partial, W^{c}\right)=\min _{\Gamma \in \hat{\partial}} \delta\left(V(\Gamma), W^{c}\right), \quad \delta\left(\phi, W^{c}\right)=\sup _{x \in W} \delta\left(x, W^{c}\right) .
$$

It is easy to check that the above norm defines a Banach space

$$
\mathscr{F}_{W}=\left\{f: \mathscr{E}\left(\mathbb{Z}^{v}\right) \rightarrow \mathbb{C}:\|f\|_{W}<\infty\right\},
$$


of contour functionals. Notice that $\mathscr{F}_{\phi}=\mathscr{F}$, where $\mathscr{F}$ is the Banach space introduced in (3.3.16) with $\xi=\exp (\tau / 8)$. In application we will choose $W$ by $\Lambda$. For any $\partial, \partial^{\prime} \in \mathscr{E}$ with $\partial \cup \partial^{\prime} \in \mathscr{E}$, it is easy to show that $\delta\left(\partial, W^{c}\right) \leqq \delta\left(\partial \cup \partial^{\prime} ; W^{c}\right)+$ $\delta\left(\partial \mid \partial^{\prime}\right)$ has been defined in (4.1.4), and so

$$
-\delta\left(\partial \cup \partial^{\prime} ; W^{c}\right) \leqq-\delta\left(\partial, W^{c}\right)+\delta\left(\partial \mid \partial^{\prime}\right),
$$

for any $\partial, \partial \in \mathscr{E}$ with $\partial \cup \partial \in \mathscr{E}$, and any finite $W \subset \mathbb{Z}^{v}$.

Before proving Proposition 4.1.3 and Proposition 4.1.4 we need the following result:

Proposition 4.3.1. Let $\mathbf{K}$ be the operator defined in (3.3.27). Then for sufficiently large $\tau,\|\mathbf{K}\|_{W} \leqq \exp \left(-\tau\left|\Gamma_{0}\right| / 28\right)$, where $\|\mathbf{K}\|_{W}$ is the operator norm of $\mathbf{K}$ in the Banach space $\mathscr{F}_{W}$.

Proof. Assume that $\|f\|_{W} \leqq 1$. Then from the definition of $\mathbf{K}_{1}$ in (3.3.26) it follows that

$$
\begin{aligned}
\left|\left(\mathbf{K}_{1} f\right)(\partial)\right| & \leqq \exp \left(-\delta\left(\partial-\Gamma_{0}, W^{c}\right)+\tau\left|\partial-\Gamma_{0}\right| / 8\right) \\
& \leqq \exp \left(-\delta\left(\partial ; W^{c}\right)+\tau|\partial| / 8\right) \exp \left(-\tau\left|\Gamma_{0}\right| / 8\right),
\end{aligned}
$$

and so

$$
\left\|\mathbf{K}_{1}\right\|_{W} \leqq \exp \left(-\tau\left|\Gamma_{0}\right| / 8\right) \text {. }
$$

We next consider $\mathbf{K}_{2}$ defined in (3.3.18). Let $\partial \in \mathscr{E}\left(\mathbb{Z}^{v}\right)$ and $\Gamma_{0} \in \partial$ be given. For any $\partial_{1}, \partial_{1}^{\prime}, \partial_{2}^{\prime}, \partial^{\prime} \in \mathscr{E}\left(\mathbb{Z}^{v}\right)$ such that $\left(\partial-\Gamma_{0}\right) \cup \partial_{1} \cup \partial_{1}^{\prime} \cup \partial_{2}^{\prime} \cup \partial^{\prime} \in \mathscr{E}$ and $\partial_{1}, \partial_{2}^{\prime} \in \mathscr{E}\left(\operatorname{Int} \Gamma_{0}\right)$, (4.3.4) and (4.1.4) simply

$$
\begin{aligned}
-\delta\left(\left(\partial-\Gamma_{0}\right) \cup\left(\partial_{1} \cup \partial_{1}^{\prime} \cup \partial_{2}^{\prime} \cup \partial^{\prime}\right), W^{c}\right) & \leqq-\delta\left(\partial \cup \partial_{1} \cup \partial_{1}^{\prime} \cup \partial_{2}^{\prime} \cup \partial^{\prime}, W^{c}\right) \\
& \leqq-\delta\left(\partial, W^{c}\right)+\delta\left(\partial \mid \partial_{1} \cup \partial_{1}^{\prime} \cup \partial_{2}^{\prime} \cup \partial^{\prime}\right) \\
& \leqq-\delta\left(\partial, W^{c}\right)+\delta\left(\Gamma_{0} \mid \partial_{1} \cup \partial^{\prime} \cup \partial_{2}^{\prime} \cup \partial^{\prime}\right) .
\end{aligned}
$$

Since $\partial_{1}, \partial_{2}^{\prime} \in \mathscr{E}\left(\operatorname{Int} \Gamma_{0}\right)$,

$$
\delta\left(\Gamma_{0} \mid \partial_{1} \cup \partial_{1}^{\prime} \cup \partial_{2}^{\prime} \cup \partial^{\prime}\right) \leqq \delta\left(\partial_{1} \mid \partial_{1}^{\prime} \cup \partial_{2}^{\prime} \cup \partial^{\prime}\right) \leqq \delta\left(\partial_{1} \mid \partial_{1}^{\prime}\right)+\delta\left(\partial_{1} \mid \partial_{2}^{\prime}\right)+\delta\left(\partial_{1}^{\prime} \cup \partial_{2}^{\prime} \mid \partial^{\prime}\right),
$$

and so

$$
\begin{aligned}
-\delta\left(\left(\partial-\Gamma_{0}\right) \cup\left(\partial_{1} \cup \partial_{1}^{\prime} \cup \partial_{2}^{\prime} \cup \partial^{\prime}\right), W^{c}\right) \leqq & -\delta\left(\partial, W^{c}\right)+\delta\left(\partial_{1} \mid \partial_{1}^{\prime}\right) \\
& +\delta\left(\partial_{1} \mid \partial_{2}^{\prime}\right)+\delta\left(\partial_{1}^{\prime} \cup \partial_{2}^{\prime} \mid \partial^{\prime}\right)
\end{aligned}
$$

We first show that for sufficiently large $\tau$,

$$
\begin{aligned}
& \sum_{\substack{\hat{o}_{1} \cup \hat{\sigma}_{2}^{\prime} \in \mathcal{E}\left(\operatorname{Int} \Gamma_{0}\right) \cdot \phi \neq \hat{\sigma}_{1}^{\prime} \in \delta^{\prime}\left(\mathbb{Z}^{v} \backslash V\left(\Gamma_{0}\right)\right) \\
\hat{\partial}_{1} \neq \phi}} \int d \omega K\left(\partial_{1} ; \omega\right) \\
& \cdot\left(\sup _{\omega \in \Omega\left(\hat{\sigma}_{1}^{\prime} \cup \hat{\sigma}_{2}^{\prime}\right)}\left|\bar{H}\left(\partial_{1}, \partial_{1}^{\prime} ; \omega\right) G\left(\partial_{1}, \partial_{2}^{\prime}: \omega\right)\right|\right) e^{\tau\left|\partial_{1}\right| / 8} \\
& \cdot \exp \left(\delta\left(\partial_{1} \mid \partial_{1}^{\prime}\right)+\delta\left(\partial_{1} \mid \partial_{2}^{\prime}\right)-\tau\left|\partial_{1}^{\prime} \cup \partial_{2}^{\prime}\right| / 8\right) \leqq \exp \left(\tau\left|\Gamma_{0}\right| / 12\right) .
\end{aligned}
$$

Note that $\delta\left(\partial_{1} \mid \partial_{2}^{\prime}\right)-\tau\left|\partial_{2}^{\prime}\right| / 8 \leqq \sum_{\Gamma^{\prime} \in \sigma_{2}^{\prime}}\left\{\delta\left(\partial_{1}, \Gamma^{\prime}\right)-\tau\left|\Gamma^{\prime}\right| / 24\right\}$ for $\tau / 12>1$. From 
(4.2.9), (4.2.12) and the definition of $G\left(\partial_{1}, \partial_{2}^{\prime} ; \omega\right)$ in (3.3.10), it follows that

$$
\sum_{\partial_{2}^{\prime}} \sup _{\omega}\left|G\left(\partial_{1}, \partial_{2}^{\prime} ; \omega\right)\right| \exp \left(\delta\left(\partial_{1} \mid \partial_{2}^{\prime}\right)-\tau\left|\partial_{2}^{\prime}\right| / 8\right) \leqq \exp \left(\tau\left|\partial_{1}\right| / 24\right)
$$

and so by (4.2.13) and Proposition 4.2.5(a) the expression in (4.3.7) is bounded by

$$
\begin{aligned}
& \sum_{\phi \neq \hat{o}_{1} \in \mathscr{\sigma}^{\prime}\left(\operatorname{In} t \Gamma_{0}\right)} \sum_{\partial_{1}^{\prime} \in \mathscr{E}\left(\mathbb{Z}^{\nu} \backslash V\left(\Gamma_{0}\right)\right)} \int_{\partial_{1}} d \omega K\left(\partial_{1} ; \omega\right) \exp \left(\tau\left|\partial_{1}\right| / 6\right) \\
& \quad \sup _{\omega}\left|\bar{H}\left(\partial_{1}, \partial_{1}^{\prime} ; \omega\right)\right| \exp \left(\delta\left(\partial_{1} \mid \partial_{1}^{\prime}\right)-\tau\left|\partial_{1}^{\prime}\right| / 8\right) \leqq \exp \left(\tau\left|\Gamma_{0}\right| / 12\right) .
\end{aligned}
$$

This proves the bound in (4.3.7).

From the definition of $\mathbf{K}_{2}$ in (3.3.18) and the relation in (4.3.6) it follows that

$$
\begin{aligned}
& \left\|\mathbf{K}_{2}\right\|_{W} \leqq \sum_{\substack{\phi \neq \hat{o}_{1} \cup \partial_{2}^{\prime} \in \mathscr{E}\left(\operatorname{Int} \Gamma_{0}\right) \\
\hat{o}_{1} \neq \phi \neq \hat{o}_{1}^{\prime} \in \mathscr{E}\left(\mathbb{Z}^{v} \backslash V(\hat{c})\right)}} \int_{\hat{\sigma}_{1}} d \omega \int_{\hat{c}_{2}^{\prime}} d \omega \int_{\hat{\sigma}_{1}^{\prime}} d \omega \\
& \cdot K\left(\partial_{1} ; \omega\right)\left|\bar{H}\left(\partial_{1}, \partial_{1}^{\prime} ; \omega\right) G\left(\partial_{1}, \partial_{2}^{\prime} ; \omega\right)\right| \\
& \sum_{\hat{o}^{\prime} \in \mathscr{E}\left(\mathbb{Z}^{v} \backslash \vee\left(\left(\hat{o}-\Gamma_{0}\right) \cup \hat{o}_{1} \cup \hat{o}_{1}^{\prime} \cup \hat{o}_{2}^{\prime}\right)\right)}\left|A\left(\partial^{\prime},\left(\partial_{1}^{\prime} \cup \partial_{2}^{\prime} ; \omega\right)\right)\right| \\
& \cdot \exp \left(\delta\left(\partial_{1} \mid \partial_{1}^{\prime}\right)+\delta\left(\partial_{1} \mid \partial_{2}^{\prime}\right)+\delta\left(\partial_{1}^{\prime} \cup \partial_{2}^{\prime} \mid \partial^{\prime}\right)\right) \\
& \cdot \exp \left(\tau\left|\partial_{1} \cup \partial_{1}^{\prime} \cup \partial_{2}^{\prime} \cup \partial^{\prime}\right| / 8-\tau\left|\Gamma_{0}\right| / 8\right) .
\end{aligned}
$$

We now use Proposition 4.2.5(b) and the bound in (4.3.7) (in that order) to obtain from (4.3.9) that

$$
\left\|\mathbf{K}_{2}\right\|_{W} \leqq \exp \left(\tau\left|\Gamma_{0}\right| / 12-\tau\left|\Gamma_{0}\right| / 8\right) \leqq \exp \left(-\tau\left|\Gamma_{0}\right| / 24\right),
$$

for sufficiently large $\tau$.

We next consider $\mathbf{K}_{3}$ defined in (3.3.19). Employing the method similar to that used to obtain (4.3.10), and Lemma 4.2.1 (and the method similar to that to obtain (4.3.13) below), it is not hard to check that

$$
\begin{aligned}
\left\|\mathbf{K}_{3}\right\|_{W} & \leqq \sum_{\Gamma \in\left[\Gamma_{0}\right]} \exp \left(-\tau\left|\Gamma_{0}\right| / 24-\tau|\Gamma| / 8\right) \\
& \leqq\left|\Gamma_{0}\right| \exp \left(-\tau\left|\Gamma_{0}\right| / 24\right) \leqq \exp \left(-\tau\left|\Gamma_{0}\right| / 25\right),
\end{aligned}
$$

for sufficiently large $\tau$. Here we have used the fact that card $\left(\left\{\Gamma \in\left[\Gamma_{0}\right]:|\Gamma|=n\right\}\right) \leqq$ $\left(\left|\Gamma_{0}\right|+n\right) \exp (a n)$ by Lemma 4.2.1 and the definition of $\left[\Gamma_{0}\right]$ in (3.3.2).

Finally, consider $\mathbf{K}_{4}$ defined in (3.3.22). Note that for $\Gamma \in\left[\Gamma_{0}\right]$,

$$
\begin{aligned}
-\delta\left(\left(\partial-\Gamma_{0}\right) \cup \Gamma \cup \partial^{\prime}, W^{c}\right) & \leqq-\delta\left(\partial \cup \Gamma \cup \partial^{\prime}, W^{c}\right) \leqq-\delta\left(\partial, W^{c}\right)+\delta\left(\partial \mid \Gamma \cup \partial^{\prime}\right) \\
& \leqq-\delta\left(\partial, W^{c}\right)+\delta\left(\Gamma_{0} \mid \Gamma \cup \partial^{\prime}\right) \\
& \leqq-\delta\left(\partial, W^{c}\right)+|\Gamma|+\delta\left(\Gamma \mid \partial^{\prime}\right) .
\end{aligned}
$$

Using (4.3.12), Proposition 4.2.5(b) and Lemma 4.2.1, it is easy to check that

$$
\left\|\mathbf{K}_{4}\right\|_{W} \leqq \exp \left(-\tau\left|\Gamma_{0}\right| / 26\right)
$$

for sufficiently large $\tau$. Combining (4.3.5), (4.3.10), (4.3.11) and (4.3.13) we proved the Proposition completely. 
We are now ready to show Proposition 4.1.3 and Proposition 4.1.4. We will use a method similar to that used in the proof of Proposition 2.1 in Sect. 8 of Chapter 2 in [18].

Proof of Proposition 4.1.3. It is obvious that Proposition 4.1.3 is a consequence of Proposition 4.1.4.

Proof of Proposition 4.1.4. To prove the proposition, first we consider $\mathbf{K}$ as an operator on the Banach space $\mathscr{F}=\mathscr{F}_{W=\phi}$. Since $\|\mathbf{K}\|_{\phi}<1$ by Proposition 4.3.1. and $\|\mathbf{1}\|_{\phi}=1, g$ is defined as the solution of the equation

$$
g=\mathbf{1}+\mathbf{K} g .
$$

That is,

On the other hand

$$
g=\sum_{n=0}^{\infty} \mathbf{K}^{n} 1
$$

$$
g_{\Lambda}=\mathbf{1}+\chi_{\Lambda} \mathbf{K} \chi_{\Lambda} g_{\Lambda}
$$

by (3.3.28), and

$$
g-g_{\Lambda}=g-\chi_{\Lambda} g+\chi_{\Lambda} g-g_{\Lambda}
$$

From (4.3.14) and (4.3.16) it follows that

$$
\chi_{\Lambda} g-g_{\Lambda}=\chi_{\Lambda} \mathbf{K}\left(g-\chi_{\Lambda} g\right)+\chi_{\Lambda} \mathbf{K}\left(\chi_{\Lambda} g-g_{\Lambda}\right) .
$$

Thus $\chi_{\Lambda} g-g_{\Lambda}$ is again a fixed point and

$$
\chi_{\Lambda} g-g_{\Lambda}=\sum_{n=0}^{\infty}\left(\chi_{\Lambda} \mathbf{K}\right)^{n} \eta_{\Lambda}
$$

where

$$
\eta_{\Lambda} \equiv \chi_{\Lambda} \mathbf{K}\left(g-\chi_{\Lambda} g\right)
$$

By Proposition 4.3.1 the series in (4.3.19) converges in any of the Banach space $\mathscr{F}_{A}$ is finite.

We next estimate $\eta_{\Lambda}$. Observe that

$$
\left(g-\chi_{\Lambda} g\right)\left(\partial^{\prime \prime}\right)=\left\{\begin{array}{lll}
0 & \text { if } & \partial^{\prime \prime} \in \mathscr{E}(\Lambda) \\
g\left(\partial^{\prime \prime}\right) & \text { if } & \partial^{\prime \prime} \notin \mathscr{E}(\Lambda) .
\end{array}\right.
$$

If $\partial^{\prime \prime} \notin \mathscr{E}(\Lambda)$, then there exist at least one contour $\Gamma^{\prime \prime} \in \partial^{\prime \prime}$ such that $d\left(\Gamma^{\prime \prime}, \Lambda^{c}\right) \leqslant 1$, which implies $\delta\left(\Gamma^{\prime \prime}, \Lambda^{c}\right) \leqslant 1$. Write

$$
\eta_{\Lambda}(\partial)=\sum_{i=1}^{4} \eta_{\Lambda, i}, \quad \eta_{\Lambda, i}=\chi_{\Lambda} \mathbf{K}_{i}\left(g-\chi_{\Lambda} g\right), \quad i=1,2,3,4 .
$$

From the definition of $\mathbf{K}_{1}$ in (3.3.26), and (4.3.21), it follows that for any $\partial \in \mathscr{E}(\Lambda)$,

$$
\eta_{\Lambda, 1}(\partial)=\left(g-\chi_{\Lambda} g\right)\left(\partial-\Gamma_{0}\right)=0
$$

Next, we consider $\eta_{\Lambda, 2}(\partial)$. We write

$$
\eta_{\Lambda, 2}(\partial)=\sum_{\partial^{\prime \prime}} \mathbf{K}_{2}\left(\partial ; \partial^{\prime \prime}\right) g\left(\left(\partial-\Gamma_{0}\right) \cup \partial^{\prime \prime}\right)
$$


where $\partial^{\prime \prime}=\partial_{1} \cup \partial_{1}^{\prime} \cup \partial_{2}^{\prime} \cup \partial^{\prime},\left(\partial-\Gamma_{0}\right) \cup \partial^{\prime \prime} \notin \mathscr{E}(\Lambda)$ and the kernel $\mathbf{K}_{2}\left(\partial ; \partial^{\prime \prime}\right)$ is defined according to the expression in (3.3.18). Since $\left(\partial-\Gamma_{0}\right) \cup \partial^{\prime \prime} \notin \mathscr{E}(\Lambda), \delta\left(\left(\partial-\Gamma_{0}\right) \cup\right.$ $\left.\partial^{\prime \prime}, \Lambda^{c}\right) \leqslant 1$, and so by (4.3.6),

$$
-1 \leqslant-\delta\left(\partial, \Lambda^{c}\right)+\delta\left(\partial_{1} \mid \partial_{1}^{\prime}\right)+\delta\left(\partial_{1} \mid \partial_{2}^{\prime}\right)+\delta\left(\partial_{1}^{\prime} \cup \partial_{2}^{\prime} \mid \partial^{\prime}\right) .
$$

Thus by (4.3.24) and (4.3.25) $\eta_{\Lambda, 2}$ is bounded by

$$
\begin{aligned}
\left|\eta_{\Lambda, 2}(\partial)\right| \leqslant & \|g\|_{\phi} e^{-\left|\Gamma_{0}\right| / 8} e^{-\partial\left(\hat{\partial}, \Lambda^{c}\right)+1} \sum_{\hat{\partial}^{\prime \prime}=\hat{\partial}_{1} \cup \hat{\partial}_{1}^{\prime} \cup \partial_{2}^{\prime} \cup \partial^{\prime}}\left|\mathbf{K}_{2}\left(\partial ; \partial^{\prime \prime}\right)\right| \\
& \cdot \exp \left(\delta\left(\partial_{1} \mid \partial_{1}^{\prime}\right)+\delta\left(\partial_{1} \mid \partial_{2}^{\prime}\right)+\delta\left(\partial_{1}^{\prime} \cup \partial_{2}^{\prime} \mid \partial^{\prime}\right)-\tau\left|\partial^{\prime \prime} \cup \partial\right| / 8\right) .
\end{aligned}
$$

Compare the above expression with that in (4.3.9). Thus repeating the argument that gave the result in (4.3.10), we obtain

$$
\left|\eta_{\Lambda, 2}(\partial)\right| \leqslant e^{-\delta\left(\theta, \Lambda^{c}\right)+\tau \mid \partial / 8}\|g\|_{\phi} e^{1-\tau\left|\Gamma_{0}\right| / 24} .
$$

Employing the method similar to that used above and repeating the arguments used to obtain (4.3.11) and (4.3.13) one may obtain

$$
\left|\eta_{\Lambda, 3}(\partial)\right|+\left|\eta_{\Lambda, 4}(\partial)\right| \leqslant e^{-\delta\left(\hat{\partial}, \Lambda^{c}\right)+\tau|\hat{\mid}| / 8}\|g\|_{\phi} 2 e^{1-\tau\left|\Gamma_{0}\right| / 26} .
$$

Thus for sufficiently large $\tau$, (4.3.23), (4.3.26) and (4.3.27) imply

$$
\left\|\eta_{A}\right\|_{\Lambda} \leqslant e^{-\tau \mid \Gamma_{0} / / 28}
$$

and so by (4.3.19) and Proposition 4.3.1 we concluded that

$$
\left\|\chi_{A} g-g_{A}\right\|_{A} \leqslant\left(1-\|\mathbf{K}\|_{A}\right)^{-1}\left\|\eta_{A}\right\|_{A}<1
$$

for sufficiently large $\tau$. The above bound, (4.3.17) and (4.3.21) imply the proposition directly.

\subsection{Sketch of the Proof of Cluster Property}

Because of notational complications involved, the detailed proof of Theorem 4.1.2 would be very lengthy. Thus we will give an outline of the proof and leave technical details to the reader.

We first describe the procedure of the proof we follow. For any $\partial, \partial^{\prime} \in \mathscr{E}\left(\mathbb{Z}^{v}\right)$ with $\partial \cup \partial^{\prime} \in \mathscr{E}\left(\mathbb{Z}^{v}\right)$, let

$$
\rho_{\mathbb{Z}^{v} \backslash V\left(\hat{o}^{\prime}\right)}(\partial)=\lim _{\Lambda \rightarrow \mathbb{Z}^{v}} \rho_{\Lambda \backslash V\left(\hat{o}^{\prime}\right)}(\partial), \quad g_{\mathbb{Z}^{v} \backslash V\left(\hat{o}^{\prime}\right)}(\partial)=\lim _{\Lambda \rightarrow \mathbb{Z}^{v}} g_{\Lambda \backslash V\left(\hat{o}^{\prime}\right)}(\partial) .
$$

The existence of the above limit follows from the method used in the proofs of Theorem 4.1.1 and Proposition 4.1.3. Assume that for any $\partial, \partial^{\prime} \in \mathscr{E}$ with $\partial \cup \partial^{\prime} \in \mathscr{E}$,

and

$$
\rho_{\mathbb{Z}^{\vee} \backslash V\left(\theta^{\prime}\right)}(\partial)-\rho(\partial) \rightarrow 0,
$$

$$
\frac{\rho\left(\partial \cup \partial^{\prime}\right)}{\rho\left(\partial^{\prime}\right)}-\rho_{\mathbb{Z}^{\vee} \backslash V\left(\hat{o}^{\prime}\right)}(\partial) \rightarrow 0,
$$

as $d\left(\partial, \partial^{\prime}\right) \rightarrow \infty\left(\delta\left(\partial, \partial^{\prime}\right) \rightarrow \infty\right)$. Then it follows that

$$
\rho\left(\partial \cup \partial^{\prime}\right)-\rho(\partial) \rho\left(\partial^{\prime}\right)=\left[\frac{\rho\left(\partial \cup \partial^{\prime}\right)}{\rho\left(\partial^{\prime}\right)}-\rho_{\mathbb{Z}^{\vee} \backslash V\left(\partial^{\prime}\right)}(\partial)\right] \rho\left(\partial^{\prime}\right)+\left[\rho_{\mathbb{Z}^{\vee} \backslash V\left(\partial^{\prime}\right)}(\partial)-\rho(\partial)\right] \rho(\partial) \rightarrow 0
$$


as $\delta\left(\partial, \partial^{\prime}\right) \rightarrow \infty$. Hence, in order to show Theorem 4.1.2 it suffices to show (4.4.2) and (4.4.3).

Let us denote that for any $B \subset \mathbb{Z}^{v}$,

$$
[B]=\{\partial \in \mathscr{E}: d(V(\partial), B) \leqslant 1\} .
$$

From (3.2.12) and (4.4.1) it follows that

$$
\begin{aligned}
\rho(\partial) & =\sum_{\partial^{\prime \prime} \in \mathscr{E}\left(\mathbb{Z}^{v} \backslash V(\partial)\right)} \int_{\partial} d \omega A\left(\partial^{\prime \prime},(\partial ; \omega)\right) g\left(\partial \cup \partial^{\prime \prime}\right), \\
\rho_{\mathbb{Z}^{v} \backslash V\left(\partial^{\prime}\right)}(\partial) & =\sum_{\partial^{\prime \prime} \in \mathscr{E}\left(\mathbb{Z}^{v} \backslash V\left(\partial \cup \partial^{\prime}\right)\right)} \int d \omega A\left(\partial^{\prime \prime},(\partial ; \omega)\right) g_{\mathbb{Z}^{v} \backslash V\left(\partial^{\prime}\right)}\left(\partial \cup \partial^{\prime \prime}\right) .
\end{aligned}
$$

Thus

$$
\begin{aligned}
\left|\rho(\partial)-\rho_{\mathbb{Z}^{v} \backslash V\left(\hat{o}^{\prime}\right)}(\partial)\right| \leqslant & \sum_{\partial^{\prime \prime} \in\left[V\left(\partial^{\prime}\right)\right]} \int d \omega\left|A\left(\partial^{\prime \prime},(\partial ; \omega)\right)\right| g\left(\partial \cup \partial^{\prime \prime}\right) \\
& +\sum_{\partial^{\prime \prime} \in \mathscr{E}\left(\mathbb{Z}^{\nu} \backslash V\left(\partial \cup \partial^{\prime}\right)\right)} \int_{\partial} d \omega\left|A\left(\partial^{\prime \prime},(\partial ; \omega)\right)\right|\left|g\left(\partial \cup \partial^{\prime \prime}\right)-g_{\mathbb{Z}^{v} \backslash V\left(\partial^{\prime}\right)}\left(\partial \cup \partial^{\prime \prime}\right)\right| .
\end{aligned}
$$

We note that the summands in (4.4.6) are summable over $\partial^{\prime \prime} \in \mathscr{E}\left(\mathbb{Z}^{v}\right)$ (by Theorem 4.1.1). Thus the first term in the right-hand side of (4.4.6) tends to zero as $\delta\left(\partial, \partial^{\prime \prime}\right) \rightarrow \infty$. In fact, using Lemma 4.2.3 and Proposition 4.2.5(b) one may show that the first term in (4.4.6) is bounded by $c \exp \left(-\delta\left(\partial, \partial^{\prime}\right)\right)$. Thus in order to proof (4.4.2) one need to show that for any given $\partial \in \mathscr{E}\left(\mathbb{Z}^{v} \backslash V\left(\partial^{\prime}\right)\right)$,

$$
g(\partial)-g_{\mathbb{Z}^{v} \backslash V\left(\partial^{\prime}\right)}(\partial) \rightarrow 0,
$$

as $\delta\left(\partial, \partial^{\prime}\right) \rightarrow \infty$.

To show (4.4.7) we use the method similar to that used in the proof of Proposition 4.1.4. Following the steps in (4.3.14)-(4.3.20) one may show that (4.4.7) holds if

$$
\left\|\chi_{\mathbb{Z}^{v} \backslash V\left(\hat{o}^{\prime}\right)} \mathbf{K}\left(g-\chi_{\mathbb{Z}^{v} \backslash V\left(\hat{o}^{\prime}\right)} g\right)\right\|_{\mathbb{Z}^{v} \backslash V\left(\hat{o}^{\prime}\right)}<\infty
$$

holds. Using the fact that

$$
\left(g-\chi_{\mathbb{Z}^{\nu} \backslash V\left(\partial^{\prime}\right)} g\right)(\partial)=\left\{\begin{array}{lll}
0 & \text { if } & \partial \in \mathscr{E}\left(\mathbb{Z}^{\nu} \backslash V\left(\partial^{\prime}\right)\right. \\
g(\partial) & \text { if } & \partial \in\left[V\left(\partial^{\prime}\right)\right],
\end{array}\right.
$$

and the method similar to that used to obtain (4.3.28), one can show that (4.4.8) holds. This proves (4.4.7) and so (4.4.2).

Finally we consider (4.4.3). For a given $\partial^{\prime} \in \mathscr{E}(\Lambda)$, denote

$$
Z\left(\Lambda ; \partial^{\prime}\right)=\sum_{\partial \in \mathscr{E}\left(\Lambda \backslash V\left(\partial^{\prime}\right)\right)} \int_{\partial} d \omega \int_{\partial^{\prime}} d \omega e^{-H\left(\partial \cup \partial^{\prime}, \omega\right)},
$$

and

$$
\tilde{g}_{\Lambda, \partial^{\prime}}(\partial)=\left[Z\left(\Lambda \backslash V(\partial) ; \partial^{\prime}\right) \prod_{\Gamma \in \partial} Z(\operatorname{Int} \Gamma)\right] / Z\left(\Lambda ; \partial^{\prime}\right) .
$$

Using the method to derive the cluster expansion (3.2.10) (and (4.4.5)) we obtain 


$$
\begin{aligned}
\frac{\rho_{\Lambda}\left(\partial \cup \partial^{\prime}\right)}{\rho_{\Lambda}\left(\partial^{\prime}\right)}= & \sum_{\left.\partial^{\prime \prime} \in \mathscr{E}\left(\Lambda \backslash V\left(\partial \cup \partial^{\prime}\right)\right)\right)} \int d \omega A\left(\partial^{\prime \prime},(\partial ; \omega)\right) \tilde{g}_{\Lambda, \hat{o}^{\prime}}\left(\partial \cup \partial^{\prime \prime}\right) \\
& +\sum_{\partial^{\prime \prime} \in \mathscr{E}(\Lambda \backslash V(\partial)): \partial} \int_{\partial} d \omega A\left(\partial^{\prime \prime},(\partial ; \omega)\right) \\
& \cdot\left[Z\left(\Lambda \backslash V(\partial) ; \partial^{\prime} \backslash \partial^{\prime \prime}\right) \prod_{\Gamma \in \partial \cup\left(\partial^{\prime \prime} \backslash \partial^{\prime}\right)} Z(\operatorname{Int} \Gamma)\right] / Z\left(\Lambda ; \partial^{\prime}\right) .
\end{aligned}
$$

Again it is easy to check that the summands in the above expression are absolutely summable over $\partial^{\prime \prime} \in \mathscr{E}\left(\mathbb{Z}^{v}\right)$, uniformly in $\Lambda$. Thus the second term in the right-hand side of (4.4.11) tends to zero uniformly in $\Lambda$ as $\delta\left(\partial, \partial^{\prime}\right) \rightarrow \infty$. In fact, it can be shown that the second term is bounded by $c \exp \left(-\delta\left(\partial, \partial^{\prime}\right)\right)$. By comparing the second expression of (4.4.5) and the first term of (4.4.11) (in the limit as $\Lambda \rightarrow \mathbb{Z}^{v}$ ) one concludes that (4.4.3) holds if for any given $\partial \in \mathscr{E}^{\mathscr{N}}\left(\mathbb{Z}^{v} \backslash V(\partial)\right)$,

$$
\tilde{g}_{\hat{o}^{\prime}}(\partial)-g_{\mathbb{Z}^{v} \backslash V\left(\hat{o}^{\prime}\right)}(\partial) \rightarrow 0,
$$

as $\delta\left(\partial, \partial^{\prime}\right) \rightarrow \infty$, where

$$
\tilde{g}_{\hat{o}^{\prime}}(\partial)=\lim _{\Lambda \rightarrow \mathbb{Z}^{v}} g_{\Lambda, \partial^{\prime}}(\partial) .
$$

The above limit exists by the method used in the proof of Proposition 4.1.3.

To prove (4.4.12), one uses the method in Sect. 3.3. to develop an integral equation for $\tilde{g}_{\Lambda, \sigma^{\prime}}$ to obtain

$$
\tilde{g}_{\Lambda, 0^{\prime}}=\mathbf{1}+\chi_{\Lambda \backslash V\left(\hat{o}^{\prime}\right)} \mathbf{K} \chi_{\Lambda \backslash V\left(\hat{o}^{\prime}\right)} \tilde{g}_{\Lambda, \hat{o}^{\prime}}+\chi_{\Lambda} \overline{\mathbf{K}} \chi_{\Lambda} \bar{g}_{\Lambda, \hat{o}^{\prime}}
$$

where $\bar{g}_{\Lambda, \partial}$ is a functional in $\mathscr{F}$ bounded by

$$
\left|\bar{g}_{\Lambda, \partial^{\prime}}\right| \leqq \exp \left(\tau\left|\partial \cup \partial^{\prime}\right| / 12\right)
$$

and $\overline{\mathbf{K}}, \overline{\mathbf{K}}=\overline{\mathbf{K}}_{2}+\overline{\mathbf{K}}_{3}+\overline{\mathbf{K}}_{4}$, is an operator obtained from the definitions of $\mathbf{K}_{2}, \mathbf{K}_{3}$ and $\mathbf{K}_{4}$ by replacing the sum over $\partial_{1}^{\prime} \in \mathscr{E}(\Lambda \backslash V(\partial))$ by the sum over $\partial_{1}^{\prime} \in \mathscr{E}(\Lambda \backslash V(\partial))$ with the restriction $\partial_{1}^{\prime} \cap \partial^{\prime} \neq \phi$. Thus it can be shown that

$$
\|\overline{\mathbf{K}}\|_{V\left(\hat{o}^{\prime}\right)}<\infty,
$$

and so

$$
\left(\chi_{\Lambda} \overline{\mathbf{K}} \chi_{\Lambda} \bar{g}_{\Lambda, \sigma^{\prime}}\right)(\partial) \rightarrow 0
$$

uniformly in $\Lambda$ as $\delta\left(\partial, \partial^{\prime}\right) \rightarrow \infty$. Since by (4.4.13)

$$
\left(\tilde{g}_{\Lambda, 0^{\prime}}-g_{\Lambda \backslash V\left(\hat{o}^{\prime}\right)}\right)(\partial)=\chi_{\Lambda} \overline{\mathbf{K}} \chi_{\Lambda} \bar{g}_{\Lambda, o^{\prime}}(\partial),
$$

(4.4.14) and (4.4.15) imply (4.4.12). Thus we have proved (4.4.3). We leave the technical details to the reader.

\section{Appendix. Proof of Proposition 4.2.2}

Proof of Proposition 4.2.2. In this Appendix we prove Proposition 4.2.2.

(a) As before we denote that for any given $B \subset \mathbb{Z}^{v}$

$$
B_{s}=\left\{x \in B: \operatorname{dist}\left(x, B^{c}\right)>s\right\}, \quad\left(B^{c}\right)_{s}=\left\{x \in B^{c}: \operatorname{dist}(x, B)>s\right\} .
$$


Then from the definition of contours in Sect. 2.1 and the regularity assumption in assumption 2.3.2 it follows that

$$
\sup _{\omega} e^{\delta\left(\Gamma, \Gamma^{\prime}\right)}\left|\Phi_{2}\left(\Gamma, \Gamma^{\prime} ; \omega\right)\right| \leqq 2 \sum_{\substack{x \in V(\Gamma)_{s} \\ y \in V\left(\Gamma^{\prime}\right)_{s}}} J(\|x-y\|),
$$

and so the expression in (4.2.1) is bounded by

$$
2 \sum_{\Gamma \in \partial} \sum_{\Gamma^{\prime} \in \partial^{\prime}} \sum_{\substack{x \in V(\Gamma)_{s} \\ y \in V\left(\Gamma^{\prime}\right)_{s}}} J(\|x-y\|) \leqq \min \begin{cases}2 \sum_{x \in B_{s}} \sum_{y \in\left(B^{c}\right)_{s}} & J(\|x-y\|) \\ 2 \sum_{x \in\left(V(\partial)_{s}\right)} \sum_{y \in\left(V(\partial)^{c}\right)_{s}} J(\|x-y\|) . & \end{cases}
$$

Let us estimate the first expression in (A.3). The number of sites in $B_{s}$ having distance $n$ from bd $\left(B_{s}\right)$ in less than $\left|\mathrm{bd}\left(B_{s}\right)\right|$. Thus the first expression in (A.3) is bounded by

$$
2\left|\operatorname{bd}\left(B_{s}\right)\right| \sum_{n=0}^{\infty} \sum_{y \in \mathbb{Z}^{v}} J(2 s+n+\|y\|) .
$$

Similarly the second expression in (A.3) is bounded by

$$
2\left|\operatorname{bd}\left(V(\partial)_{s}\right)\right| \sum_{n=0}^{\infty} \sum_{y \in \mathbb{Z}^{v}} J(2 s+n+\|y\|),
$$

(A.4) and (A.5) and (2.3.7) imply part (a) of the proposition.

(b) By the regularity condition (2.3.5) the expression in (4.2.2) is bounded by

$$
2 \sum_{\Gamma^{\prime} \in \mathscr{G}\left(\mathbb{Z}^{\nu} \backslash V(\Gamma)\right)} \sum_{\substack{x \in V(\Gamma)_{s} \\ y \in V\left(\Gamma^{\prime}\right)_{s}}} J(\|x-y\|) e^{-\tau\left|\Gamma^{\prime}\right| / 48}
$$

For a given site $x \in V(\Gamma)_{s}$, let $y\left(\Gamma^{\prime}\right)$ be the site in $V\left(\Gamma^{\prime}\right)_{s}$ with $\left\|x-y\left(\Gamma^{\prime}\right)\right\|=$ $\operatorname{dist}\left(x, V\left(\Gamma^{\prime}\right)_{s}\right)$. Then it follows that for given $x \in V(\Gamma)_{s}$ and $\Gamma^{\prime} \in \mathscr{C}\left(\mathbb{Z}^{v} \backslash V(\Gamma)\right)$,

$$
\sum_{y \in V\left(\Gamma^{\prime}\right)_{s}} J(\|x-y\|) \leqq\left|V\left(\Gamma^{\prime}\right)\right| J\left(\left\|x-y\left(\Gamma^{\prime}\right)\right\|\right) .
$$

For given $x \in B \subset \mathbb{Z}^{v}$, let

$$
\mathscr{D}_{x, y}=\left\{\Gamma^{\prime} \in \mathscr{C}\left(\mathbb{R}^{v}\right): \operatorname{dist}\left(x, V\left(\Gamma^{\prime}\right)\right)=\|x-y\|\right\} .
$$

Then by (A.7) and Lemma 4.2.1 the expression in (A.6) is bounded by

$$
\begin{aligned}
& 2 \sum_{x \in V(\Gamma)_{s}} \sum_{y \in\left(V(\Gamma)^{c}\right)_{s}} J(\|x-y\|) \sum_{\Gamma^{\prime} \in \mathscr{D}_{x, y}}\left|V\left(\Gamma^{\prime}\right)\right| e^{-\tau\left|\Gamma^{\prime}\right| / 48} \\
& \quad \leqq 2 \sum_{x \in V(\Gamma)_{s}} \sum_{y \in\left(V(\Gamma)^{c}\right)_{s}} J(\|x-y\|) \\
& \quad \leqq 2|\operatorname{bd}(V(\Gamma))| \sum_{n=0}^{\infty} \sum_{y \in \mathbb{Z}^{v}} J(2 s+n+\|y\|),
\end{aligned}
$$

for sufficiently large $\tau$. Thus by (2.3.7) and the above bound we proved the part (b) of the proposition. 
(c) By (2.3.5) the expression in (4.2.3) is bounded by

$$
2 \sum_{\Gamma \in \mathscr{C}(B)} \sum_{\Gamma^{\prime} \in \hat{\delta}} \sum_{\substack{x \in V(\Gamma)_{s} \\ y \in V\left(\Gamma^{\prime}\right)_{s}}} J(\|x-y\|) e^{-\tau|\Gamma| / 48} \leqq 2 \sum_{\Gamma \in \mathscr{C}(B)} \sum_{x \in V(\Gamma)_{s}} \sum_{y \in\left(B^{c}\right)_{s}} J(\|x-y\|) e^{-\tau|\Gamma| / 48} \text {. }
$$

For a given finite $B \subset \mathbb{Z}^{v}$, let

$$
\mathscr{D}_{r}=\{\Gamma \in \mathscr{C}(B): \operatorname{dist}(V(\Gamma), \mathrm{bd}(B)) \leqq r\} .
$$

Then by Lemma 4.2.1, the expression in (A.8) is bounded by

$$
2 \sum_{r=0}^{\infty} \sum_{y \in \mathbb{Z}^{v}} J(2 s+r+\|y\|) \sum_{\Gamma \in \mathscr{Q}_{r}} e^{-\tau|\Gamma| / 48}|\Gamma| \leqq 2 \sum_{r=0}^{\infty} \sum_{y \in \mathbb{Z}^{v}} J(2 s+r+\|y\|)|\mathrm{bd}(B)| .
$$

Part (c) of the proposition follows from (2.3.7) and the above bound.

(d) Again by (2.3.5) the expression in (4.3.4) is bounded by

$$
\begin{aligned}
& 2 \sum_{\Gamma \in \mathscr{C}(B)} \sum_{\Gamma^{\prime} \in \mathscr{G}\left(\mathbb{Z}^{v} \backslash B\right)} \sum_{\substack{x \in V(\Gamma)_{s} \\
y \in V\left(\Gamma^{\prime}\right)_{s}}} J(\|x-y\|) e^{-\tau\left|\Gamma \cup \Gamma^{\prime}\right| / 48} \\
& \leqq \sum_{r=0}^{\infty} \sum_{y \in \mathbb{Z}^{v}} J(2 s+r+\|y\|) \sum_{\Gamma \in \mathscr{D}_{r}}|\Gamma| e^{-\tau|\Gamma| / 48} \sum_{\substack{\Gamma^{\prime} \in \mathscr{G} ; \\
y \in V\left(\Gamma^{\prime}\right)}} e^{-\tau\left|\Gamma^{\prime}\right| / 48} \\
& \quad \leqq 2|\operatorname{bd}(B)| \sum_{r=0}^{\infty} J(2 s+r+\|y\|),
\end{aligned}
$$

for sufficiently large $\tau$. Here we have used Lemma 4.2.1 to get the last inequality. The part $(\mathrm{d})$ of the proposition follows from (2.3.7) and the above bound. This proved Proposition 4.2.2 completely.

Acknowledgements. I am very grateful to the referee for his criticism leading to a clear version of the paper, and to the Korean Science Foundation for financial support.

\section{References}

1. Bricmont, J., Kuroda, K., Lebowitz, J. L.: First order phase transitions in lattice and continuous systems: Extension of Pirogov-Sinai theory. Commun. Math. Phys. 101, 501-538 (1985)

2. Dobrushin, R. L.: Existence of a phase transition in two- and three-dimensional models. Theory Probab. Appl. 10, 193 (1965)

3. Dobrushin, R. L., Zahradnik, M.: Phase diagrams for the continuous spin models: Extension of Pirogov-Sinai theory. Preprint

4. Fröhlich, J.: The pure phase (harmonic functions) of generalized processes. Or: Mathematical physics of phase transitions and symmetry breaking. Bull. Am. Math. Soc. 84, No. 2, 165-193 (1978)

5. Fröhlich, J., Israel, R., Lieb, E. H., Simon, B.: Phase transitions and reflection positivity, I and II. Commun. Math. Phys. 62, 1-34 (1978) and J. Stat. Phys. 22, 297-347 (1980)

6. Fröhlich, J., Lieb, E. H.: Phase transitions in anisotropic lattice systems. Commun. Math. Phys. 60, 233-267 (1978)

7. Fröhlich, J., Simon, B., Spencer, T.: Infrared bounds, phase transitions and continuous symmetry breaking. Commun. Math. Phys. 50, 79-85 (1976)

8. Griffiths, R. B.: Peierls's proof of spontaneous of a two-dimensional Ising ferromagnet. Phys. Rev. A. 136, 193 (1965)

9. Kotecky, R., Preiss, D.: An inductive approach to Pirogov--Sinai theory. Proc. Winter School on Abstract Analysis 1983. Suppl. Ai. Rend Circ. Mat. Palermo (1983) 
10. Kotecky, R., Preiss, D.: Cluster expansion for abstract polymer models. Commun. Math. Phys. 103, 491-498 (1986)

11. Malyshev, V. A.: Cluster expansions in lattice models of statistical physics and the quantum theory of fields. Russ. Math. Surv. 35, 1-62 (1980)

12. Park, Y. M.: Cluster expansion for classical and quantum lattice system. J. Stat. Phys. 27, No. 3, 553-576 (1983)

13. Park, Y. M.: Extension of the Pirogov-Sinai theory of phase transitions to infinite range interactions II. Phase diagram. Commun. Math. Phys. 114, 219-241 (1988)

14. Peierls, R.: On Ising's model of ferromagnetism. Proc. Camb. Philos. Soc. 32, 477 (1936)

15. Pirogov, S. A., Sinai, Ya. G.: Phase diagram of classical lattice systems I and II. Theor. Math. Phys. 25, 1185 (1976) and 26, 39 (1976)

16. Ruelle, D.: Statistical mechanics: Rigorous results. New York: Benjamin 1969

17. Seiler, E.: Gauge theories as a problem of constructive quantum field theory and statistical mechanics. Lecture Notes in Physics, Vol. 159. Berlin, Heidelberg, New York: Springer 1982

18. Sinai, Ya. G.: Theory of phase transition: Rigorous results. New York: Pergamon Press 1982

19. Zahradnik, M.: An alternative version of Pirogov-Sinai theory. Commun. Math. Phys. 93, 559 (1984)

Communicated by J. Fröhlich.

Received February 26, 1987; in revised form May 28, 1987

Note added in proof. It has been realized that one has to multiply the factor $1 /\left(\left|\left[\Gamma_{0}\right] \cap \hat{o}^{\prime}\right|+1\right)$ to the summand in the second term in the right hand side of (3.3.3). Thus each summand appeared in the summations $\sum_{\Gamma \in\left[\Gamma_{0}\right]} \sum_{\hat{\sigma}^{\prime}}$ in Section 3.3 must also be multiplied by the above factor. Since the factor is bounded by 1 , no changes are needed in the rest of the paper. 